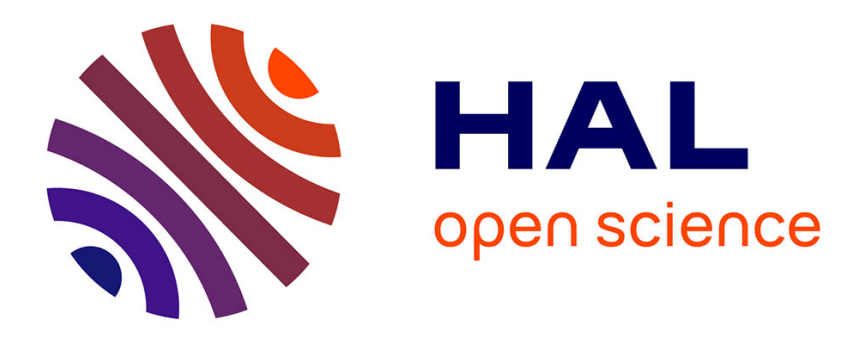

\title{
Field and Numerical Investigation of Transport Mechanisms in a Surface Storage Zone
}

Jorge Sandoval, Emmanuel Mignot, Luca Mao, Pablo Pastén, Diogo Bolster, Cristian Escauriaza

\section{- To cite this version:}

Jorge Sandoval, Emmanuel Mignot, Luca Mao, Pablo Pastén, Diogo Bolster, et al.. Field and Numerical Investigation of Transport Mechanisms in a Surface Storage Zone. Journal of Geophysical Research: Earth Surface, 2019, 124 (4), pp.938-959. 10.1029/2018JF004716 . hal-02381346

\section{HAL Id: hal-02381346 https://hal.science/hal-02381346}

Submitted on 26 Nov 2019

HAL is a multi-disciplinary open access archive for the deposit and dissemination of scientific research documents, whether they are published or not. The documents may come from teaching and research institutions in France or abroad, or from public or private research centers.
L'archive ouverte pluridisciplinaire HAL, est destinée au dépôt et à la diffusion de documents scientifiques de niveau recherche, publiés ou non, émanant des établissements d'enseignement et de recherche français ou étrangers, des laboratoires publics ou privés. 


\section{JGR Earth Surface}

\section{RESEARCH ARTICLE \\ 10.1029/2018JF004716 \\ Field and Numerical Investigation of Transport Mechanisms in a Surface Storage Zone}

Key Points:

- Large-scale turbulent coherent structures are the fundamental mechanisms of transport between surface storage zones (lateral recirculation areas) and the main channel in rivers

- A combination of field measurements and 3-D numerical simulations provides a better understanding of the flow field and transport in the recirculation zone - Three global mass transport models are used to study the exchange processes and represent the temporal evolution of solute concentration

Correspondence to:

C. Escauriaza,

cescauri@ing.puc.cl

Citation:

Sandoval, J., Mignot, E., Mao, L., Pastén, P., Bolster, D., \& Escauriaza, C. (2019). Field and numerical investigation of transport mechanisms in a surface storage zone. Journal of Geophysical Research: Earth Surface, 124, 938-959. https://doi.org/10.1029/2018JF004716

Received 13 APR 2018 Accepted 15 FEB 2019 Accepted article online 28 FEB 2019 Published online 6 APR 2019

CC2019. American Geophysical Union. All Rights Reserved.

\author{
Jorge Sandoval ${ }^{1}$ (D) , Emmanuel Mignot ${ }^{1,2}$ iD , Luca Mao $^{3,4}$ iD, Pablo Pastén ${ }^{1,5}$, Diogo Bolster ${ }^{6}$, \\ and Cristián Escauriaza ${ }^{1,4}$ iD
}

${ }^{1}$ Departamento de Ingeniería Hidráulica y Ambiental, Pontificia Universidad Católica de Chile, Santiago, Chile, ${ }^{2}$ Univ Lyon, INSA Lyon, CNRS, LMFA UMR5509, Villeurbanne, France, ${ }^{3}$ School of Geography, University of Lincoln, Lincoln, UK, ${ }^{4}$ Centro de Investigación para la Gestión Integrada de Desastres Naturales (CIGIDEN), Macul, Chile, ${ }^{5}$ Centro de Desarrollo Urbano Sustentable (CEDEUS), Providencia, Chile, ${ }^{6}$ Department of Civil and Environmental Engineering and Earth Sciences, University of Notre Dame, Notre Dame, IN, USA

\begin{abstract}
In-stream surface storage zones (SSZs) caused by lateral recirculation areas play a significant role in the transport and fate of contaminants in rivers. Lateral recirculating areas have long residence times that favor nutrient uptake, accumulation of pollutants, and interactions with reactive sediments. In watersheds affected by acid-mine drainage, SSZs have profound effects on biogeochemical processes, controlling the local concentration and distribution of toxic elements along the channel. Despite the importance of turbulent flow dynamics on these processes, limited work has been carried out to analyze mass transport in natural SSZs with complex geometries. In this investigation we study a SSZ in the Lluta River, located in a high-altitude environment in northern Chile, by coupling field measurements and 3-D numerical simulations to understand the transport mechanisms with the main channel. We measure the velocity field using an acoustic Doppler velocimeter (ADV) and large-scale particle image velocimetry (LSPIV), extracting the bathymetry from digital image processing. Using these data, we perform detached-eddy simulations (DES) to analyze the mean flow, turbulence statistics, and the dynamics of large-scale coherent structures. From this detailed description of the turbulent flow, we study the mass exchange and the time evolution of the mean concentration of a passive scalar in the SSZ by testing three upscaled models: a classical linear transport model, a two-storage formulation, and a fractional transport model. The analysis integrates temporal and spatial scales to provide a new perspective on the turbulent flow in SSZs and their effects on global mass transport in rivers.
\end{abstract}

\section{Introduction}

The complex hydrodynamics that are generated by flow interactions with geomorphic features in rivers (i.e., confluences, bars, riffles, pools, flow obstructions, meander bends, etc.) have significant effects on biogeochemical processes and nutrient cycles, which maintain the properties and spatial distribution of fluvial ecosystems (Jackson et al., 2012; Khosronejad et al., 2016; Patil et al., 2013). Energetic, large-scale, unsteady, coherent structures are continuously generated by local shear, pressure imbalances, and flow separation, and they exert a fundamental control on transport and deposition of contaminants along the channel (Mignot et al., 2016, 2017).

In watersheds impacted by mining activities, rivers usually transport dissolved pollutants and toxic particles that degrade the water quality, increasing stresses on aquatic environments (Butler et al., 2008; Guerra, Gonzalez, et al., 2016; Hudson-Edwards, 2003). The contamination from mining is either produced by direct discharges or by acid drainage from abandoned legacy tailing deposits in sites where they have accumulated for decades.

Recent events, such as the Gold King Mine spill of 2015 in the Animas River watershed in Colorado (Rodriguez-Freire et al., 2016) and tailing dam failures in Brazil, Spain, the United Kingdom, Bolivia, and Chile (Hudson-Edwards, 2016; Kossoff et al., 2014), have shown the damaging effects of acid mine drainage, lowering the $\mathrm{pH}$ and increasing the concentration of metals in water and sediment. Catastrophic spills can also have long-term environmental impacts, as the contaminants are mobilized along the river and interact with natural processes, downstream ecosystems, and human activities in the watershed, affecting agricul- 
ture and water supplies. The deposition and accumulation of mine tailings in river channels have also been shown to amplify flood hazards near ephemeral streavms in arid regions, which are subject to rapid floods (Wilcox et al., 2016).

In recent years, we have investigated in detail the effects of legacy mining in the high-altitude environment of the Lluta River in northern Chile, focusing on the coupling of physical and biogeochemical processes in the system (Abarca et al., 2017; Arce et al., 2017; Contreras et al., 2015; Guerra, Gonzalez, et al., 2016; Guerra, Simonson, et al., 2016; Leiva et al., 2014). Acid drainage in this watershed has increased the concentration of elements and compounds such as arsenic, aluminum, iron, chlorides, and sulfates in the river. The most remarkable interaction of the flow hydrodynamics with geochemical processes associated with acid drainage is the formation and transport of solid reactive particles that control the physical and chemical fate of arsenic in the watershed (Contreras et al., 2015; Guerra, Gonzalez, et al., 2016). As acid drainage mixes with the basic stream, it is neutralized, triggering the chemical precipitation of iron and aluminum oxyhydroxides. These highly reactive particles have a strong binding capacity toward metals and metalloids, actively changing their chemical speciation from the dissolved phase to the solid phase. The speciation processes in the river and their implications have been widely discussed in our previous investigations (for details, the reader is referred to Abarca et al., 2017; Arce et al., 2017; Guerra, Gonzalez, et al., 2016; Guerra, Simonson, et al., 2016).

Turbulent coherent structures that are produced by geomorphic features in this channel constitute the underlying physical mechanisms that control the fate of acid-mine drainage pollutants and their interactions with the fluvial ecosystem, especially at in-stream surface storage zones (SSZs). These lateral recirculating areas, due to their lower velocities and long residence times, can form local repositories of arsenic-rich sediments. The dynamics of the flow in SSZs have a large-scale (i.e., at large temporal and spatial scales) impact on the chemical reactivity of the pollutants, favoring interactions with hyporheic zones (Knapp et al., 2017), which produces changes in the downstream water quality when contaminants are released by resuspension or desorption processes (Butler et al., 2008; Guerra, Gonzalez, et al., 2016). Vortex interactions and large-scale velocity fluctuations play a significant role on mass exchange between the SSZ and the main channel, ultimately affecting nutrient cycling and stream connectivity (Jackson et al., 2012; Mignot et al., 2016; O'Connor et al., 2010).

Lateral recirculation zones commonly occur downstream of flow obstructions and can create an arcuate recession of the bank line referred to as a lateral cavity. In general, the flow that develops in open-channel lateral cavities has three characteristic features: (1) shearing and flow separation at the leading edge in the upstream corner (detachment point); (2) a self-sustained turbulent mixing that develops from the detachment point and impinges at a point downstream (reattachment point); and (3) a recirculating region within the cavity located between the streambank and the mixing layer composed of one or more counterrotating structures (Jackson et al., 2013; Mignot et al., 2016; Schmidt, 1990).

Specifically, the mechanisms that drive all these processes begin at the upstream corner with the vortex shedding emerging from the shear layer between the SSZ and the main channel. In this section of the flow, high-frequency small-scale vortex structures are advected downstream in an unsteady process. These structures coalesce and grow as they move forward as Kelvin-Helmholtz-type instabilities produced by the interaction of the recirculating flow and the mixing layer vortices (Jackson et al., 2015). When they reach the reattachment point, the vortices impinge on the trailing edge, causing pressure fluctuations and unsteady transport of vorticity into the downstream cavity region (Chang et al., 2006; Jackson et al., 2013, 2015). Finally, the entrained vortices travel as a jet-like flow along the downstream wetted perimeter of the cavity, causing the flow to recirculate. In addition to these phenomena, other processes develop at different time scales, such as the low-frequency flapping of the entire shear layer (alternating positive and negative transverse velocity fluctuations) and the formation of secondary vortices within the cavity, generally induced by interactions with the wall (Jackson et al., 2013; Liu \& Katz, 2013; Mignot et al., 2016; Schmidt, 1990).

In idealized rectangular geometries, the number of vortices that form in the recirculating region is determined by the geometrical aspect ratio $(W / L)$, defined as the ratio of the cavity width normal to the flow direction $(W)$ and cavity length at the interface parallel to the main flow direction ( $L$; Constantinescu et al., 2009; Jackson et al., 2013, 2015; Mignot et al., 2016; Weitbrecht \& Jirka, 2001). Most previous work on cavity flows has focused on two-dimensional flow patterns in rectangular geometries, where the aspect ratio and the Reynolds number strongly determine the presence of secondary vortices (Mignot et al., 2016). 
Jackson et al. (2013) reported that secondary structures increase the mean residence time due to their slower circulation velocities and longer exchange time scales. By contrast, complex realistic geometries induce different recirculating patterns (Jackson et al., 2013; Ozalp et al., 2010), generating a strong three-dimensional (3-D) flow, with more complex dynamics, as the shear layer and columnar vortical structures interact with the channel morphology.

Previous experimental and numerical investigations have studied mass exchange between SSZs and open-channel flows (e.g., Chang et al., 2006; Constantinescu et al., 2009; Jackson et al., 2012; Mignot et al., 2016, 2017; Ozalp et al., 2010; Weitbrecht \& Jirka, 2001; Weitbrecht et al., 2008). These analyses have focused on the calculation of dispersion relations (Jackson et al., 2015) and exchange coefficients for transient storage models (Bencala \& Walters, 1983; Constantinescu et al., 2009; Jackson et al., 2012; O'Connor et al., 2010; Weitbrecht et al., 2008), which are parametrized as functions of dimensionless numbers and geometrical properties of the lateral cavities (Drost et al., 2014; Jackson et al., 2013).

To determine the residence times in SSZs, Jackson et al. (2012) performed numerical simulations in a natural lateral cavity using a 3-D Reynolds-averaged Navier-Stokes (RANS) formulation. They utilized the results and field observations to develop a conceptual model that they called a box one-dimensional (1-D) approach to characterize the mass exchange. Jackson et al. (2013) also derived empirical equations based on nondimensional parameters of the flow to estimate the mean residence time in SSZs, considering the bed roughness in natural conditions.

Despite the importance of the dynamics of turbulent flows in SSZs and their consequences on contaminant transport that can affect the entire watershed, most models of mass exchange with lateral recirculating flows have focused on idealized or simplified configurations (Drost et al., 2014; Jackson et al., 2015; Kimura \& Hosoda, 1997; Tuna et al., 2013), with the exception of the studies carried out by Jackson et al. (2012). Improving our understanding of the large-scale dynamics of coherent structures in realistic SSZs with natural geometries is thus critical to identify the connections of physical processes across scales and to determine the fundamental mechanisms of transport in the channel and their interactions with morphology.

Understanding the dynamics of the unsteady and highly 3-D coherent structures is also important for improving global analyses of SSZs that are typically carried out using 1-D transient storage models to characterize the mass exchange with the main channel (O'Connor et al., 2010).

The objective of this investigation is to understand the physical mechanisms that control transport between a natural SSZ and the river and to evaluate their large-scale effects on contaminant transport. In this case we select a prominent SSZ in the Lluta River, downstream of the confluence where the arsenic-laden particles are formed. Through measurements in the field and numerical simulations, we focus on the full 3-D dynamics of the flow to provide new insights on turbulent coherent structures that exist in a natural channel, as well as their global effects on mass transport.

We conducted measurements to characterize the hydrodynamics of the flow in the main channel and the SSZ and performed 3-D numerical simulations to resolve the large-scale dynamics of the coherent structures of the flow in the SSZ and in the adjacent section of the main channel, identifying the salient mechanisms of transport that influence the fate of the contaminants in the river (Alvarez et al., 2017). The simulations of these flows are also particularly challenging due to the highly complex natural geometry of the riverbed and the intricate emergent structure of the turbulent flow within the SSZ, involving many energetic and interacting large-scale coherent structures.

By resolving such features in field measurements and numerical simulations, a detailed characterization of the spatiotemporal evolution of mass transport can be achieved, enabling the development and testing of novel upscaled models for large-scale transport that incorporate processes typically missed by traditional approaches. In this work we will ask what kind of upscaled model can capture emergent behaviors.

The paper is organized as follows: Sections 2 and 3 contain the description of the study site and field techniques and the detached-eddy simulation (DES) model for the numerical simulations, respectively. In section 4 , we present the field measurements and the numerical simulation results, including details of the time-averaged flow field, turbulence statistics, and the dynamics of instantaneous coherent structures. We later perform an upscaling analysis of the global mass transport using three different 1-D models in section 5. Finally, in section 6, we present the conclusions, summarize our findings and outline topics for future research. 


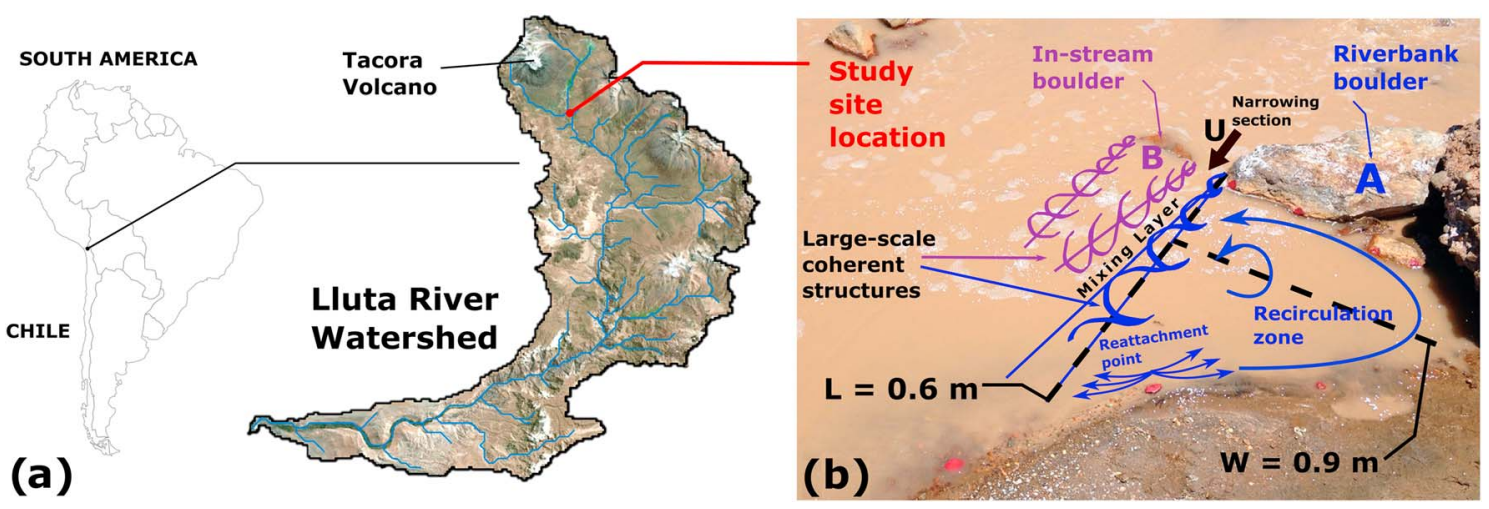

Figure 1. (a) Location of the Lluta river watershed and the study site. (b) Schematic representation of the flow features developed in the selected surface storage zone. The length and width of the cavity are indicated. Point A represents the riverbank boulder, while point B corresponds to the in-stream boulder hereinafter for all figures.

\section{Field Measurements}

\subsection{Study Site}

The Altiplano is a semiarid, high-altitude volcanic plateau located between the eastern and western cordillera of central Andes, between $15^{\circ}$ and $28^{\circ} \mathrm{S}$ (Laity, 2008). The increase of water demand for agricultural production, industrial uses, energy generation, and water supply has motivated a growing interest in the water resources of these extreme high-Andean environments. Although the water demand is increasing, this region is characterized by limited rainfall ( $<310 \mathrm{~mm} / \mathrm{year}$ ), low humidity, strong solar radiation, and high evaporation rates $(4.9 \pm 0.5 \mathrm{~mm} /$ day; Contreras et al., 2015). Rainfall in the Altiplano exhibits a considerable seasonal and interannual variability, and it is mainly concentrated from December to February, when more than $70 \%$ of the precipitation occurs (Garreaud et al., 2003).

Our study site is a river reach of the Lluta River, located approximately 1,000 m downstream of the confluence between Azufre and Caracarani rivers ( $17^{\circ} 50^{\prime} 20.48^{\prime \prime} \mathrm{S}, 69^{\circ} 42^{\prime} 25.75^{\prime \prime} \mathrm{W}$ ), where reactive arsenic-laden particles are formed due to acid-mine drainage. The study site is located in a remote area in the mountains of the Altiplano region, at an elevation of approximately 4,000 m above sea level, in an extreme environment, with no access to electricity, drinking water, or services of any kind. We focus on a SSZ found at the downstream side of a boulder located on the left riverbank. The presence of another smaller boulder in the stream, combined with the flow obstruction of the riverbank boulder, generates a narrowing section just upstream of the SSZ. The interaction between the flow and the boulders produces a jet-like flow, where large-scale structures emerge from the narrowing section and are advected by the flow. A complex surface flow is observed in the field, characterized by a large recirculating structure in the protected area, and the impinging point (also called reattachment point) located at the left bank, where the flow divides in opposite directions: Part of the flow enters the cavity, while another fraction continues downstream in the main channel, as shown in Figure $1 b$.

We selected the cavity due to its proximity to the confluence and because it is similar in size and configuration to other boulder-created lateral recirculation zones in the channel, which are common in the area. This SSZ is therefore an interesting place to analyze mass transport phenomena due to its potential role as contaminant retention zone, contributing to the particle deposition in the protected region, and with a complex turbulent flow dynamics that control the mass and momentum exchange between the main channel and the lateral cavity.

River discharge in the main channel is $Q=0.27 \mathrm{~m}^{3} / \mathrm{s}$, and the mean water depth at the gauging section is $h=0.1 \mathrm{~m}$ with a channel width of $b=5.9 \mathrm{~m}$. Therefore, the bulk velocity in the main channel is equal to $U_{b}=Q /(b h)=0.47 \mathrm{~m} / \mathrm{s}$. With this information and considering that $b>>h$, the bulk Reynolds number of the flow is equal to $R e=U_{b} h / v=45,800$. The sampled flow conditions are 
Table 1

Bulk Flow Conditions at the Surface Storage Zone Location

\begin{tabular}{lccccc}
\hline$Q\left(\mathrm{~m}^{3} / \mathrm{s}\right)$ & $h(\mathrm{~m})$ & $b(\mathrm{~m})$ & $U_{b}(\mathrm{~m} / \mathrm{s})$ & $R e$ & $F r$ \\
\hline 0.27 & 0.1 & 5.88 & 0.47 & 45,800 & 0.48 \\
\hline
\end{tabular}

representative of flow during the majority of the year because the only changes in the hydrology of the channel occur in summer due to high plateau winter (Contreras et al., 2015), however, we recognize that they may change in response to flooding. Table 1 summarizes the flow characteristics of the study site.

\subsection{Methods}

Three methodologies were employed to obtain field data: (1) microacoustic Doppler velocimeter (ADV) 3-D velocity measurements at multiple locations within the SSZ and at the interface; (2) Large-scale Particle Image Velocimetry (LSPIV) to get 2-D surface velocities; and (3) a survey of the 3-D bed topography with high-resolution, using an image analysis technique. River discharge and water depth were also measured using a handheld ADV (Flowtracker Handheld ADV, Sontek) in a gauging section located at $2 \mathrm{~m}$ downstream of the SSZ. Table 2 lists the techniques we utilized for the flow description.

\subsection{ADV Measurements}

The ADV measurements were performed using a micro-ADV (Sontek 16-MHz instrument) with a sampling rate of $50 \mathrm{~Hz}$. The field setup for the measurements consisted of a system of rails mounted on a wooden structure that we assembled on the river, above the interface of the main channel and the SSZ. The ADV was mounted in a carriage, which ran on tracks to position the device above a specific horizontal location (see Figure 2b). We measured a total of 69 points in the region of interest, spanning five cross-stream sections distributed as shown in Figure 2a. The longitudinal position of the profiles was measured within a local coordinate system attached to the rail structure we installed in the river. This local system was georeferenced with a Differential Global Positioning System instrument (DGPS; Trimble R3 L1 GPS system) described below, to ensure consistency with the rest of the measurement techniques employed. Details of the measurement points are summarized in Table 3; at each position, we measured the velocity field for 3 min to compute the mean flow and turbulence statistics (Reynolds stresses and spectra). Despite the shorter measurement time in the field, compared to laboratory measurements with ADVs, the results show that the duration is sufficient to ensure the convergence of low-frequency peaks in the spectral analysis.

\subsection{Surface LSPIV}

We performed LSPIV, which is a technique to capture the instantaneous surface velocity field by identifying patterns formed by surface particles seeded in the flow. We employed small pieces of paper and carried out a statistical analysis of consecutive images extracted from a video recording of the free surface flow. Particle displacements between frames are calculated by computing a 2-D cross-correlation on pairs of orthorectified images, separated by a given time step (Fujita et al., 1998). The orthorectification is carried out through the measurement of a set of points that are visible on the videos, which are globally referenced at the edge of the water surface and are used to determine the spatial position of each pixel in the images. This LSPIV technique has been widely used in laboratory and field applications, particularly hydraulic studies in rivers (for details, the reader is referred to Coz et al., 2010; Fujita et al., 1998; Kantoush et al., 2011).

In this work, we recorded the video images using a commercially available digital videocamera (Sony Handycam DCR-SR45). The camera was located on the left riverbank at approximately $5 \mathrm{~m}$ above the water surface level. The movies were stored in the internal memory of the camera in MPG (Moving Picture Experts) format at a rate of 30 frames per second $(\Delta t=33.3 \mathrm{~ms})$ and with an image resolution of $720 \times 480$ pixels.

Table 2

Summary of Techniques Employed in the Field Measurements

\begin{tabular}{ll}
\hline Technique employed & \multicolumn{1}{c}{ Features for flow description } \\
\hline LSPIV & $\begin{array}{l}\text { 2-D time-averaged flow characteristics } \\
\text { on the water surface } \\
\text { ADV }\end{array}$ \\
$\begin{array}{l}\text { 3-D instantaneous flow characteristics } \\
\text { in several points of the flow }\end{array}$ \\
$\begin{array}{l}\text { High-resolution topography } \\
\text { measurements based on image analysis } \\
\text { and DGPS topographical survey }\end{array}$ & numerical simulations of the turbulent flow \\
\hline
\end{tabular}

Note. LSPIV = large-scale particle image velocimetry; ADV = acoustic Doppler velocimeter; DGPS $=$ Differential Global Positioning System. 


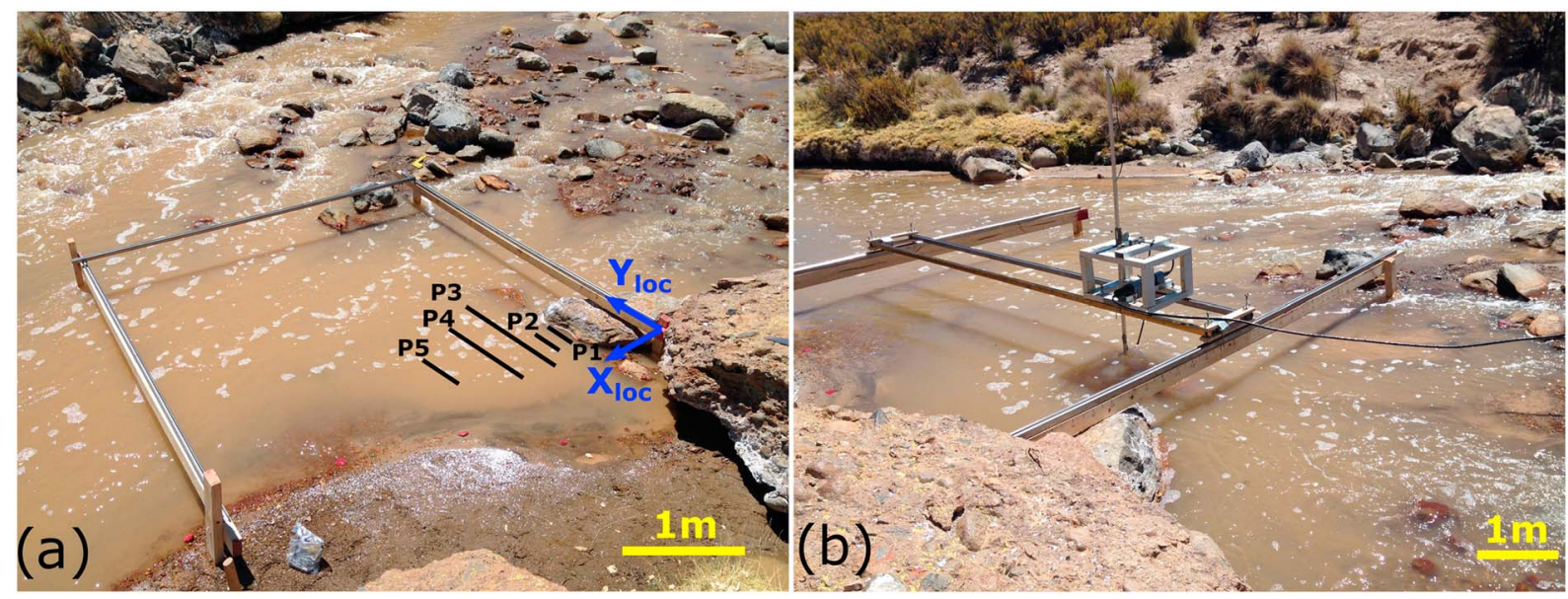

Figure 2. Field measurement setup for the ADV. (a) Rail system installed in the river to position the ADV at the measurement points, showing the five cross sections. (b) Cart with the ADV moving on the rails. ADV = acoustic Doppler velocimeter.

Seven movies of approximately 2 min were recorded over a time period of $1 \mathrm{hr}$, covering an area of approximately $4 \mathrm{~m}$ in the streamwise direction by $2 \mathrm{~m}$ in the spanwise direction. During that total time period, flow conditions of the river were constant as verified by the discharge measurements downstream. This LSPIV methodology was used to obtain the time-averaged flow field at the free surface, which was used to identify the main structures that help us describe the flow.

The topography measurements for the orthorectification process were obtained by using the DGPS device. We defined nine Ground Reference Points that were located in the vicinity of the cavity by painting pebbles red, as shown in Figure 3. The precise global coordinates of these points measured with the DGPS were reported in the UTM (Universal Transverse Mercator) system.

We selected a set of frames with high particle density to ensure a good correlation between images. Due to difficulties in providing a steady supply of tracer particles in the flow, the videos were split into sets of frames, which were used separately to obtain the mean velocity field at the free surface. In total, we used 1,954 frames, which is equivalent to $65 \mathrm{~s}$.

\subsection{Bathymetry Survey}

A digital elevation model (DEM) of the bed topography in the cavity zone was generated through the Structure from Motion (SfM) photogrammetry technique. This method, as in traditional photogrammetry, employs overlapping images taken from multiple viewpoints. The difference is that the algorithm is capable of determining the internal camera geometry, position, and orientation automatically, without the need for a predefined set of ground control visible points (Micheletti et al., 2015). This method was applied using the software Photoscan.

We surveyed the bathymetry of the area of interest by taking photographs of the dry bed. After finishing the flow measurements, we enclosed the study zone with sandbags and drained the area with a pump before collecting the images used to construct the DEM. The flow of the near-left bank of the river was deflected,

\begin{tabular}{lcc} 
Table 3 \\
Number of Points Measured With the Microacoustic Doppler Velocimeter at \\
Each Profile and the Position of the Cross Sections in the Local Coordinate \\
System \\
\hline Profile & $X_{\text {loc }}(\mathrm{m})$ & Points measured \\
\hline 1 & 0.05 & 4 \\
2 & 0.10 & 2 \\
3 & 0.30 & 32 \\
4 & 0.90 & 24 \\
5 & 1.5 & 7 \\
\hline
\end{tabular}

Note. All the points were measured at 5-cm depth. 


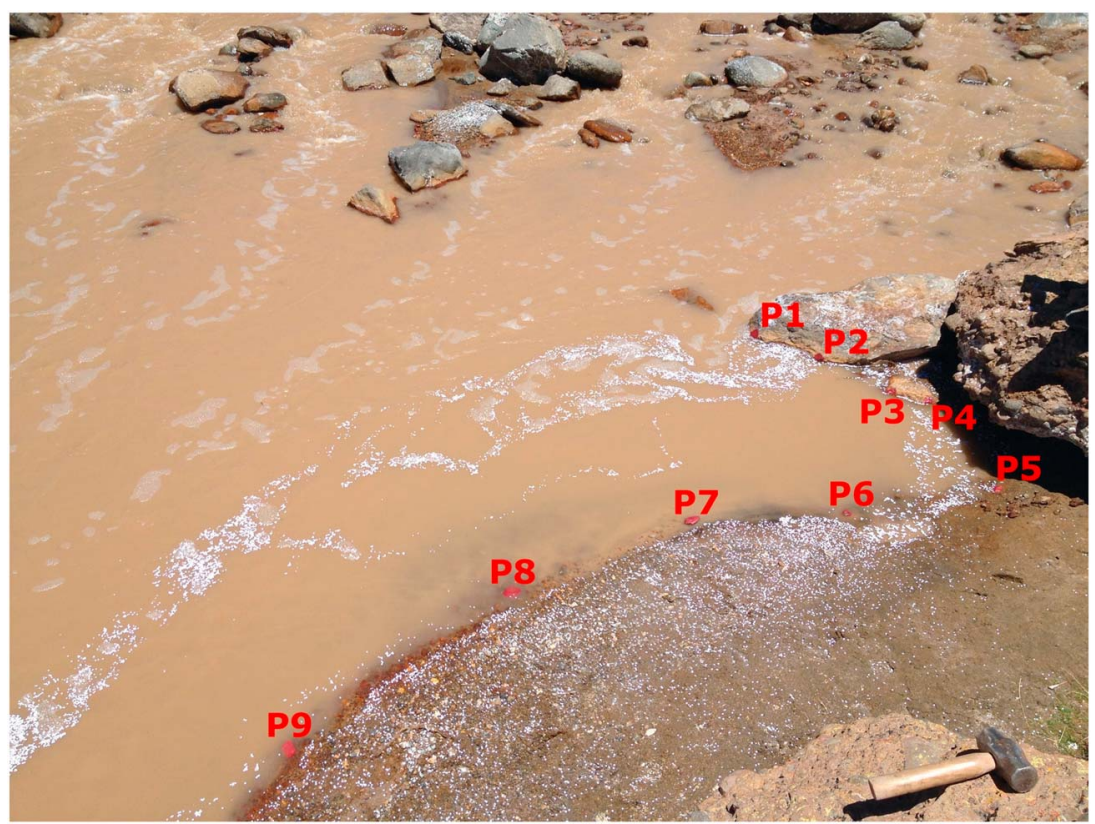

Figure 3. Points measured with the Differential Global Positioning System device to generate the Ground Reference Point in the global coordinate system that is used to perform the large-scale particle image velocimetry analysis. The camera is positioned in the video capture position for large-scale particle image velocimetry measurements.

allowing us to take multiple photos of the bed. Draining the study zone avoids refraction problems associated with SfM measurements (Dietrich, 2017).

In this case, the photographs were taken using a 16 megapixel digital camera (Canon PowerShot A4000 IS). For the coordinate referencing of the images, we employed the DGPS points that were also used for the orthorectification of the LSPIV. A set with a total of 150 pictures was used for bathymetry reconstruction.

To process the images of the bathymetry, we generated an orthorectified raster image as shown in Figure 4a. This image has a spatial resolution of $0.948 \mathrm{~cm}$ for the bed topography, which was later used as input to generate a boundary fitted curvilinear mesh for the 3-D numerical simulations of the flow in the SSZ.

With the DEM, it is also possible to determine the most relevant geometric features of the cavity. As reported previously, an important parameter to characterize the flow in the SSZ is the aspect ratio $(W / L)$. For this case, we follow the approach used by Jackson et al. (2012), defining the ratio between the maximum cavity width normal to the mean flow direction $(W)$, and cavity length at the main channel SSZ interface parallel to the mean flow direction ( $L$; see Figure $4 c$ ). For this case, the width and length measured in the field correspond to $W=0.9 \mathrm{~m}$ and $L=0.6 \mathrm{~m}$, respectively. The aspect ratio was estimated as $W / L=0.6$, which is a value in the range of the cases reported for natural streams $(W / L<1$; Jackson et al., 2012).

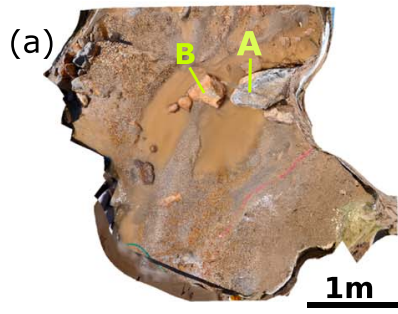

(b)

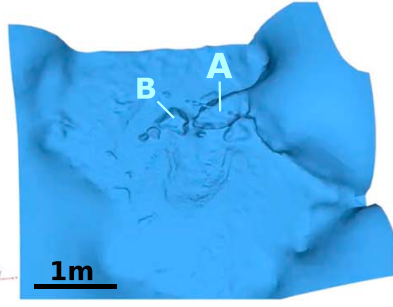

(c)

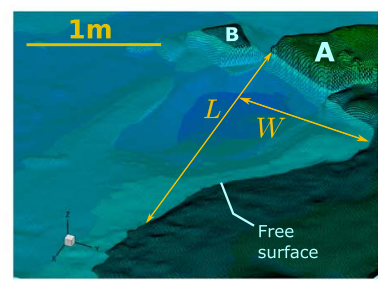

Figure 4. Processing the bathymetry from photogrammetry. (a) Orthorectified image obtained from image analysis performed with Photoscan; (b) digital elevation model generated from the orthorectified image; and (c) filtered digital elevation model used to generate the grid used in the numerical simulations. The width and length correspond to $W=0.9 \mathrm{~m}$ and $L=0.6 \mathrm{~m}$, respectively. 


\section{Numerical Model for DES}

To analyze the details of the dynamics of the turbulent flow in the SSZ, we perform 3-D coherent-structure resolving numerical simulations, to capture the energetic dynamics of the flow in the shear layer and inside the cavity, as well as the mass transport and exchange with the main channel.

The governing equations for the resolved flow are the 3-D unsteady incompressible Reynolds-averaged Navier-Stokes equations (URANS) equations for the conservation of mass and momentum, nondimensionalized by the mean water depth $(h)$ and the bulk velocity in the main channel $\left(U_{b}\right)$. The equations are transformed to a generalized curvilinear coordinate system to fit the domain to the SSZ bathymetry obtained from the photogrammetry process, and they can be written in vector format, in generalized curvilinear coordinate system and strong conservation form as follows (for details, the reader is referred to Escauriaza \& Sotiropoulos, 2011a, 2011b):

$$
\Gamma \frac{\partial Q}{\partial t}+J \frac{\partial}{\partial \xi^{j}}\left(F^{j}-F_{v}^{j}\right)=0
$$

where

$$
\begin{aligned}
\Gamma & =\operatorname{diag} \quad[0111] \\
Q & =\left[\begin{array}{ll}
P, u_{1}, u_{2}, u_{3}
\end{array}\right]^{T} \\
F^{j} & =\frac{1}{J}\left[U^{j}, u_{1} U^{j}+P \xi_{x_{1}}^{j}, u_{2} U^{j}+P \xi_{x_{2}}^{j}, u_{3} U^{j}+P \xi_{x_{3}}^{j}\right]^{T} \\
F_{v}^{j} & =\frac{1}{J}\left(\frac{1}{R e}+v_{t}\right)\left[0, g^{m j} \frac{\partial u_{1}}{\partial \xi^{m}}+R_{m 1} \xi_{x_{m}}^{j}, g^{m j} \frac{\partial u_{2}}{\partial \xi^{m}}+R_{m 2} \xi_{x_{m}}^{j}, g^{m j} \frac{\partial u_{3}}{\partial \xi^{m}}+R_{m 3} \xi_{x_{m}}^{j}\right]^{T} .
\end{aligned}
$$

In these equations, $P=p+2 / 3 k$, where $k$ is the turbulent kinetic energy (TKE) and $p$ is the pressure divided by the density. $u_{i}(i=1,2,3)$ are the Cartesian velocity components, $x_{i}$ are the Cartesian coordinates, $J$ is the Jacobian, $\xi_{x_{i}}^{j}$ are the metrics of the geometric transformation, $U^{j}=u_{i} \xi_{x_{i}}^{j}$ are the contravariant velocity components, $g^{i j}=\xi_{x_{k}}^{i} \xi_{x_{k}}^{j}$ are the components of contravariant metric tensor, $R e$ is the Reynolds number, and tensor $R_{i j}$ is defined as $R_{i j}=\left(\partial u_{j} / \partial \xi^{k}\right) \xi_{j}^{k}$.

For the turbulence model, we employed a hybrid URANS/LES (Large eddy simulation) approach known as DES (Spalart, 2009). DES is based on the one-equation Spalart-Allmaras (S-A) closure for the URANS equations, which consists on a relation for the auxiliary variable $\tilde{v}$ related to the eddy viscosity. The model can be expressed in the curvilinear coordinate system as follows:

$$
\frac{\partial \widetilde{v}}{\partial t}+J \frac{\partial}{\partial \xi^{j}}\left[F_{t}^{j}-F_{t v}^{j}\right]+J \quad H_{t}=0
$$

where

$$
\begin{aligned}
& F_{t}^{j}=\frac{1}{J}\left[U^{j} \widetilde{v}\right] \\
& F_{t v}^{j}=\frac{1}{J}\left[\frac{1}{\sigma}\left(\frac{1}{R e}+\widetilde{v}\right) g^{m j} \frac{\partial \widetilde{v}}{\partial \xi^{m}}\right] \\
& H_{t}=\frac{1}{J}\left[-c_{b_{1}}\left(1-f_{t_{2}}\right) \tilde{S} \widetilde{v}+\left(c_{w_{1}} f_{w}-\frac{c_{b_{1}}}{\kappa^{2}} f_{t_{2}}\right)\left(\frac{\widetilde{v}}{\tilde{d}}\right)^{2}-\frac{1}{\sigma} c_{b_{2}} g^{m j} \frac{\partial \widetilde{v}}{\partial \xi^{m}} \frac{\partial \widetilde{v}}{\partial \xi^{m}}\right] .
\end{aligned}
$$

In the DES approach, the S-A turbulence model functions as the subgrid scale model of LES in regions away from the wall, where the grid density can resolve the scales of fluid motion near the size of the grid spacing. The length scale of the destruction term of the S-A model is modified, using the following expression:

$$
\tilde{d}=\min \left(d, C_{\mathrm{DES}} \Delta\right),
$$

where $d$ is the distance to solid walls, and $\Delta=\max (\Delta x, \Delta y, \Delta z)$ is the largest dimension of the grid cell and the model constant is set equal to its standard value $C_{\mathrm{DES}}=0.65$ (Shur et al., 1999).

For the mass transport, we consider the volume fraction of a conservative solute, which is modeled through an advection-diffusion equation as

$$
\frac{\partial C}{\partial t}+J \frac{\partial}{\partial \xi^{j}}\left(\frac{U^{j}}{J} C\right)=J \frac{\partial}{\partial \xi^{j}}\left[\left(D+\frac{v_{t}}{\sigma_{t}}\right) \frac{g^{j k}}{J} \frac{\partial C}{\partial \xi^{k}}\right],
$$



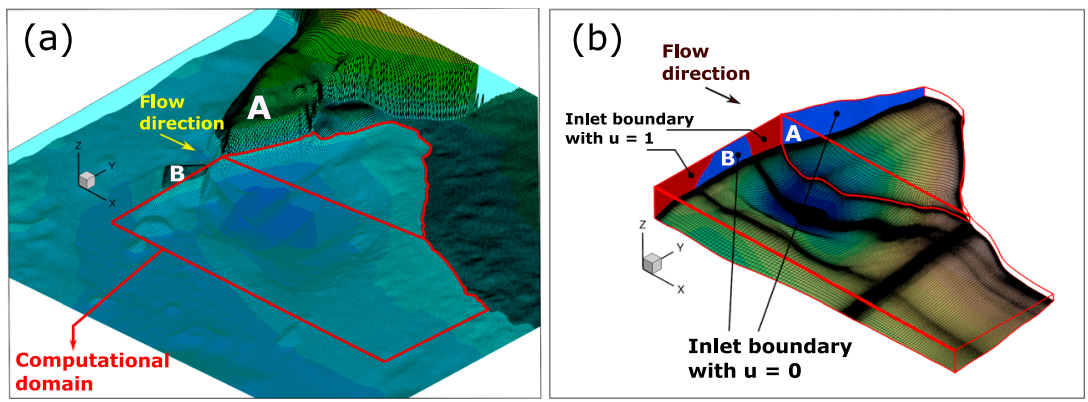

Figure 5. (a) Top view of the computational domain used for numerical simulations. (b) Bottom overset grid. The regions of interest, such as the interface between the main channel and the cavity, as well as the shear-layer reattachment zone, have finer grid resolution.

where $C$ is the nondimensional volumetric concentration of the passive scalar, $D$ is the molecular diffusion coefficient, and $\sigma_{t}$ is the turbulent Schmidt number, taken as 1.0. As discussed in our previous research for similar Reynolds numbers and grid resolutions (Escauriaza \& Sotiropoulos, 2011a), the molecular and modeled turbulent transport in the region of interest have a smaller impact, compared to the advective transport driven by the resolved large-scale turbulent coherent structures of the flow in this DES formulation. The advection-diffusion approach is commonly used to represent the transport of dissolved substances and suspended sediments (Julien, 2010), and it is here selected to model the mass transport due to the small size of the particles (2.5-500 $\mu \mathrm{m}$; Guerra, Gonzalez, et al., 2016; Leiva et al., 2014).

We solve the governing equations in generalized curvilinear coordinates using a dual-time-stepping artificial compressibility iteration scheme. The artificial compressibility form of the governing equations is discretized using a second-order-accurate finite-volume method on a nonstaggered computational grid. The advective terms are discretized using the second-order accurate, upwind biased QUICK scheme, and central differencing is employed for the pressure gradients, viscous fluxes, and source terms in the turbulence equation. For the advection-diffusion equation, we discretize the advective terms using QUICK with a flux limiter (Leonard, 1991) to avoid unphysical oscillations and the diffusive terms using a second-order central differencing scheme. The discrete equations are marched in time to advance the solution to the next time step by adopting the dual-time-stepping method. The system of equations is integrated using a pressure-based implicit preconditioner (Sotiropoulos \& Constantinescu, 1997) enhanced with local-time-stepping and V-cycle multigrid acceleration. The complex geometry of the SSZ region and the main channel is handled using domain decomposition with overset grids.

Applications and performance of this model have been discussed in great detail in a series of previous papers (e.g. Escauriaza \& Sotiropoulos, 2011a, 2011b, 2011c; Link et al., 2012; Paik et al., 2007, 2010). In all these studies, the accuracy of the numerical method was demonstrated by qualitative and quantitative comparisons with available experimental data, typically in terms of mean flow quantities and turbulence statistics.

The computational domain was obtained using the DEM generated from image analysis described in the previous section. A curvilinear structured grid was built combining the DGPS information of the bed topography and the water surface level measured in the LSPIV analysis. The computational domain was discretized using two overset grids with a total of $7.2 \times 10^{6}$ nodes, which are clustered near the solid walls using a hyperbolic tangent distribution., with $y^{+}<1.0$ (see Figure 5a). The jet-like flow formed by the boulders corresponds to the inlet boundary condition. No-slip conditions are imposed at all solid boundaries, while a zero-shear and rigid plane boundary condition is applied at the free surface and on the right side of the domain, far from the cavity (see Figure 5b). To simulate the mass transport of a passive scalar, we study the evolution of the flow by setting up a constant concentration $C=1$ inside the cavity and $C=0$ in the main channel. We prescribe this uniform concentration within the SSZ to track the solute as it is exchanged and transported in the main channel to later test large-scale 1-D models of transport. 
(a)

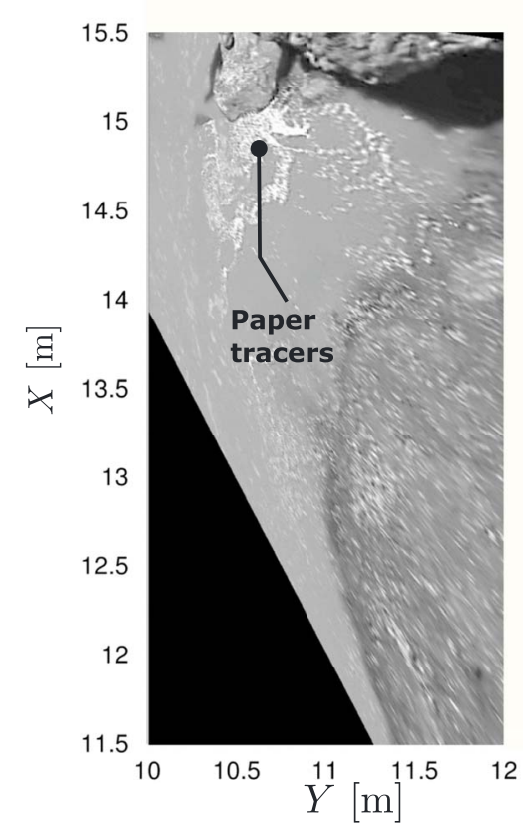

(b)

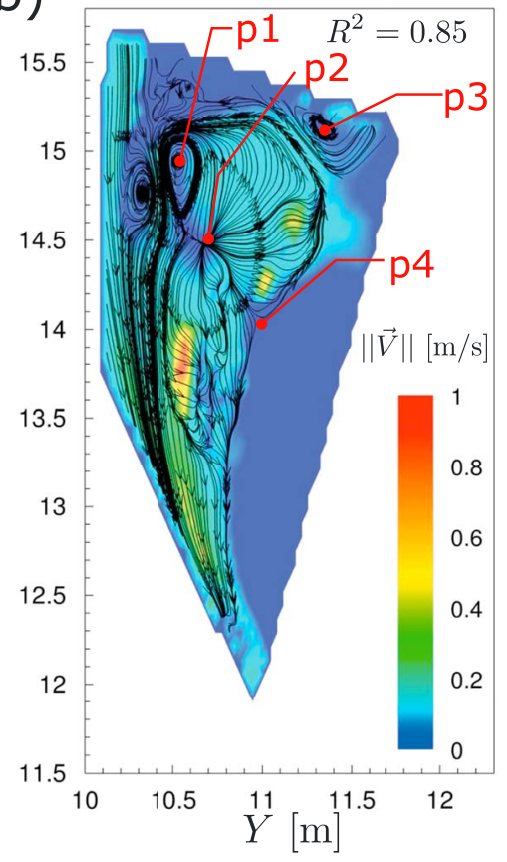

Figure 6. Mean flow field measured with LSPIV. Points p1-p4 are marked to identify the main features of the mean flow in the surface storage zone (see section 4.1 for details). The variables $X$ and $Y$ correspond to the Cartesian coordinate system defined by orthorectified images in LSPIV analysis. (a) Orthorectified image of the study zone, showing an instantaneous distribution of paper pieces; and (b) streamlines of the time-averaged flow field $(\|\vec{V}\|)$ at the water surface obtained from LSPIV measurements. The $R^{2}$ threshold used to filter the data is 0.85 . LSPIV = large-scale particle image velocimetry.

\section{Analysis of the SSZ Flow From Field Observations and Numerical Simulations}

\subsection{Time-Averaged Flow Field}

First, we provide an analysis of the time-averaged flow field that characterizes the SSZ, identifying the main structures and comparing the field data and numerical simulations. As already noted, recirculating vortical structures in the SSZ are expected, potentially with secondary and higher-order structures depending on

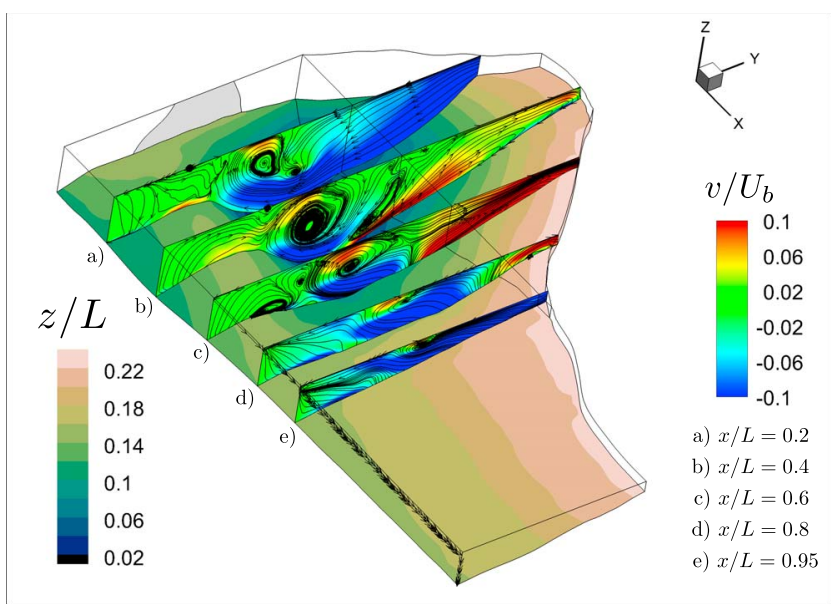

Figure 7. Five vertical transects (a-e) of the mean flow field computed with detached-eddy simulation. The slices are colored by the dimensionless transverse velocity $\left(v / U_{b}\right)$, and the streamlines are plotted to visualize the flow patterns developed between the main channel and the cavity. The bathymetry is colored by the dimensionless bed elevation $(z / L)$ 


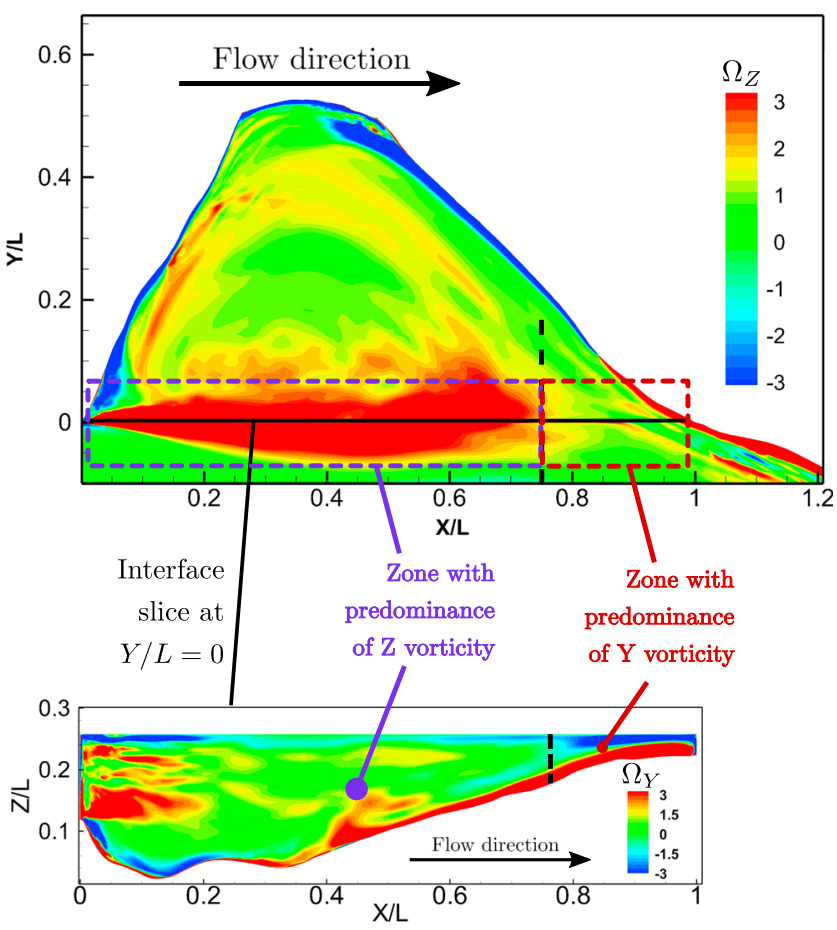

Figure 8. Computed time-averaged vorticity field $(\Omega)$. (a) Vertical vorticity ( $Z$ direction) at the free surface and (b) cross-stream vorticity ( $Y$ direction) at the interface between the main channel and the surface storage zone.

the geometry and the Reynolds number (Jackson et al., 2012, 2015; Weitbrecht \& Jirka, 2001; Weitbrecht et al., 2002) In this investigation, we seek these flow patterns via the LSPIV technique. In Figure 6, we show an orthorectified instantaneous image and the time-averaged flow with a coefficient of determination $R^{2}=0.85$. We visualize the free-surface streamlines and identify the most important flow patterns in the SSZ. In Figure 6b, we show at point $\mathrm{p} 1$ the primary vortex of the recirculating region of the cavity; at point $\mathrm{p} 2$, we observe a source point on the plane (where the flow emanates at the free surface) that we will discuss in the subsequent section. This point corresponds to an upwelling structure that demonstrates the importance of the 3-D dynamics of the flow in the cavity. At point p4, we identify the reattachment point of the shear layer, where the flow bifurcates at the trailing edge. Finally, at point p3, we observe a secondary structure, which appears as a counterrotating vortex relative to the recirculation pattern of the primary structure.

It is worth noting that capturing the source point is a challenging task, as the paper is advected quickly from this region, and the LSPIV algorithm cannot capture the velocity field as clearly. It is necessary to seed the flow with a significant concentration of particles and measure for long periods to capture this region with better precision. The 3-D unsteady mechanism that produces this flow is discussed in more detail in the following section.

The free surface flow field highlights that there exists a complex 3-D behavior within the cavity and the main channel. To elucidate in detail the mean flow in these regions, we look to the numerical simulations. In Figure 7, we show five streamwise vertical transects (perpendicular to the main flow), where we observe the main 3-D flow patterns developed at the interface between the main channel and the cavity. At the first upstream transect $(x / L=0.2)$, we identify a predominance of transverse flow from the cavity to the main channel. The second transect $(x / L=0.4)$ shows an intermediate behavior, with slow transverse velocities, while the third and fourth transects $(x / L=0.6$ and $x / L=0.8$, respectively) show a strong predominance of 


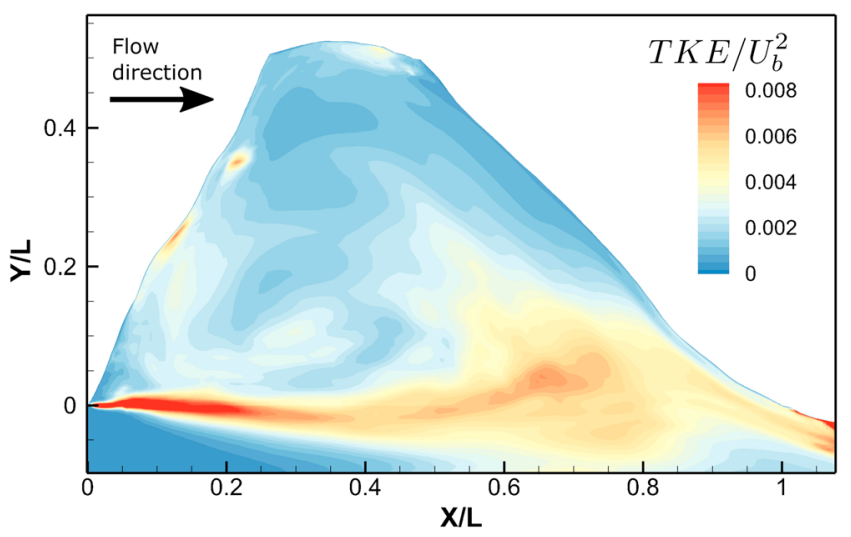

Figure 9. Resolved nondimensionalized TKE $\left(T K E / U_{b}^{2}\right)$ field at the free surface, showing the effect of the shear layer at the interface of the cavity. $\mathrm{TKE}=$ turbulent kinetic energy transverse average velocity from the main channel to the cavity. Finally, the fifth transect shows a significant transverse flow from the cavity to the main channel again. We can relate this complex 3-D behavior to the patterns observed at the free surface. In the case of the first three transects, they are strongly related to the primary vortex identified in Figure 6; in the case of the last two transects, they are clearly related to the impingement point, where the flow bifurcates. The fourth and the fifth transects capture the flow in opposite directions, showing that on average, the extent of the flow bifurcation covers the entire water column.

\subsection{Time-Averaged Vorticity Field}

In simplified geometries, $Z$-oriented vortical structures grow continuously along the mixing layer and impinge on the trailing edge of the cavity, causing the entrainment of vorticity into the cavity. These vortical structures have a vertical $Z$-orientation, and the higher vorticity region is attached to the wall of the cavity (larger vorticity values are observed along the wetted perimeter). In realistic geometries, the time-averaged flow field is highly 3-D as observed in our simulations. In Figure 8a, we plot the computed mean vertical vorticity field in the $Z$ direction at the free surface. We observe that this vorticity distribution exhibits an expanding plume along the mixing layer due to the growing vortical structures generated from the Kelvin-Helmholtz instabilities. This two-dimensional feature is the predominant condition in the vertical direction, up to distance equal to $x / L=0.75$. At this location, the adverse slope generates a significant change on the vorticity distribution, producing a pocket of high $Y$-oriented vortical structures.

Figure $8 \mathrm{~b}$ shows the mean cross-stream vorticity field (in the $Y$ direction), in a vertical plane that corresponds to the interface between the main channel and the SSZ. This vorticity distribution is very different from cases with idealized geometries. The morphology of the bed has a strong influence on the coherent structure dynamics, and their impacts are observed on the mean flow field, including the free surface. The pocket of high time-averaged $Y$ vorticity that emerges from the bed suggests a strong interaction with the wall, which lifts off the vortices and interacts with the shear-layer near the free surface, as discussed in a subsequent section.

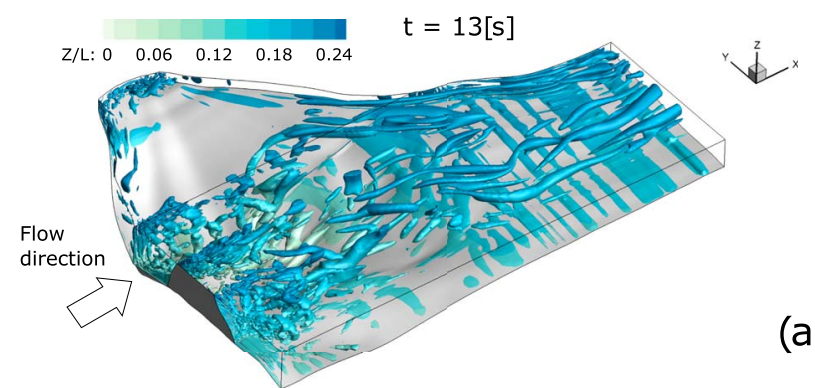

\subsection{Resolved TKE}

We also analyze the second-order statistics of the velocity field, by computing the TKE:

$$
T K E=\frac{1}{2}\left\langle u_{i}^{\prime} u_{i}^{\prime}\right\rangle
$$

where $u_{i}^{\prime}$ corresponds to the instantaneous velocity fluctuation of each velocity component. In Figure 9, we plot the TKE field at the free surface, showing the high turbulence intensity at the interface, with large magnitude upstream and lower values within the cavity. The spatial evolution of TKE exhibits a decreasing trend as it moves along the mixing layer. This decay is explained by the fact that in the upstream region, the narrowing section imposed by the boulders generates a jet-like structure and flow acceleration, inducing a vortex shedding with high-frequency velocity fluctuations. At the downstream section of the interface $(x / L>0.5)$, the bed elevation makes the flow shallower, increasing the effects of bed resistance and inducing a general trend toward lower velocities attenuating the magnitude of the TKE.

This TKE distribution also differs from experimental results for idealized geometries with similar aspect ratios (Jackson et al., 2015; Liu \& Katz, 2013), where the presence of a sharp edge at the end of the cavity produces an impingement point with a strong adverse pressure gradient and
Figure 10. Instantaneous coherent structures visualized with the $q$ criterion $(q=0.2)$ colored by depth. The images, separated by $1 \mathrm{~s}$, show the coherent-structure distribution along the mixing layer in the main channel. At the leading edge of the cavity, the vortices are smaller with a seemingly chaotic behavior, and they organize downstream in larger streamwise-oriented structures near the trailing edge. 


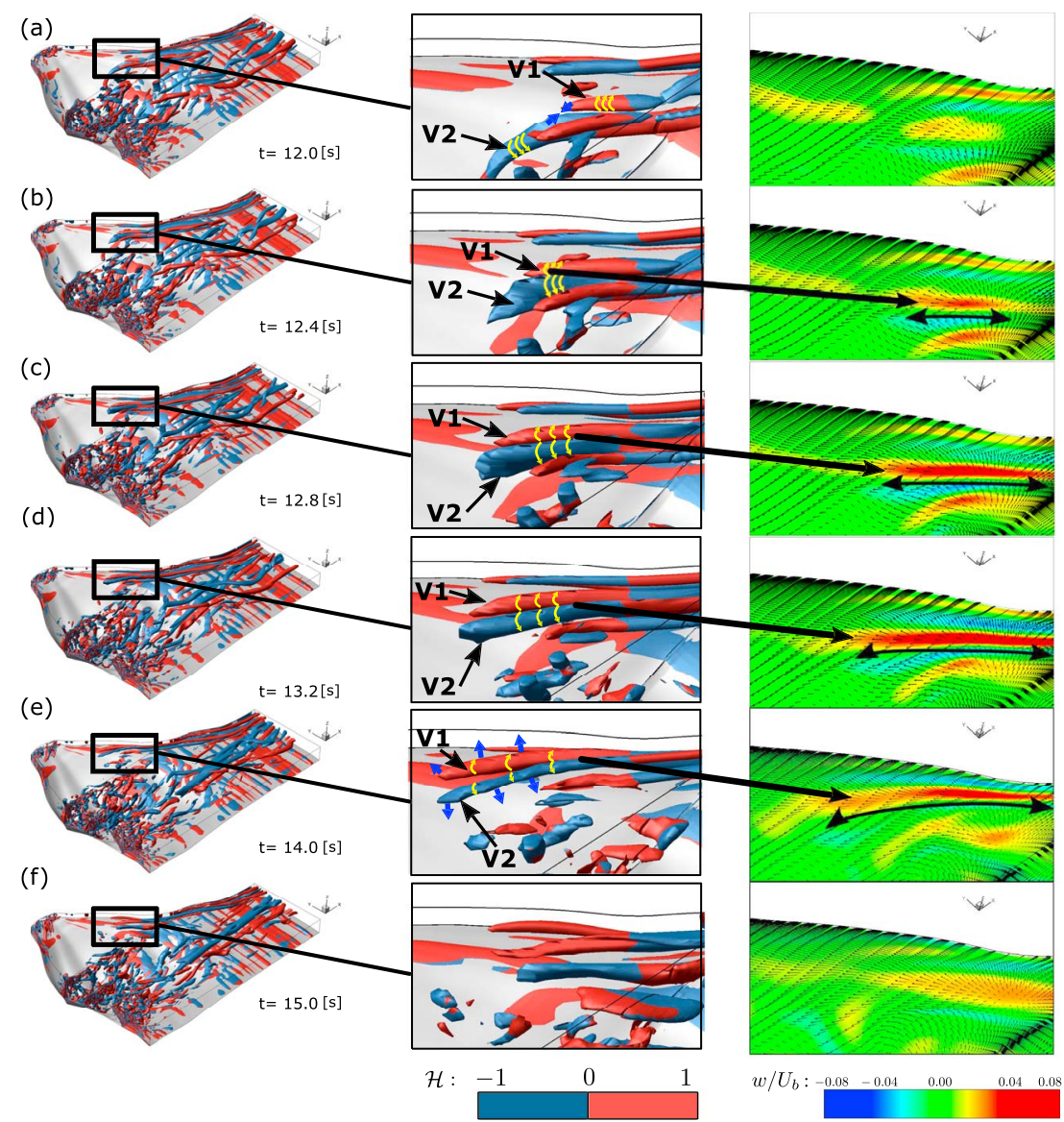

Figure 11. (a) to (f) depict a sequence of images that illustrates the interaction of instantaneous coherent structures at the trailing edge of the cavity. The vortices that emerge from the bed are stretched as they approach the free-surface, generating an upwelling event. Each rows corresponds to a specific time in the sequence. The left column shows the instantaneous coherent structures visualized with the $q$ criterion $(q=0.2)$ colored by helicity $(\mathcal{H})$. The middle column shows a zoomed visualization of the coherent structures at the trailing edge, where the upwelling events occur. The right column contains the instantaneous velocity field at the free surface colored by dimensionless vertical velocity $\left(v / U_{b}\right)$.

unsteady transport of vorticity. These features, associated with a constant water depth, produces an increase of the TKE along the mixing layer in idealized cases.

\subsection{Coherent Structure Dynamics}

The flow topology we described in the cavity region is a consequence of a complex unsteady interaction of energetic large-scale coherent structures that are continuously formed in the main channel and inside the SSZ. In this section we elucidate the dynamic mechanisms that generate these flow patterns, which control the 3-D behavior observed at the impingement zone and near the trailing edge of the cavity. To visualize the 3-D coherent dynamics of the flow, we use the $q$ criterion, computed from the calculated velocity fields, defined as follows:

$$
q=\frac{1}{2}\left(\Omega_{i j} \Omega_{i j}-S_{i j} S_{i j}\right)
$$

where $\Omega_{i j}$ and $S_{i j}$ denote the antisymmetric and symmetric parts of the velocity gradient tensor, respectively. According to Hunt et al. (1988), the large-scale vortices are visualized by the identification of areas where $q>0$, defining regions where local rotation rate is greater than the strain rate.

In Figure 10, we show instantaneous images of the 3-D structures visualized with the $q$ criterion, separated by $1 \mathrm{~s}$. We observe two main regions of the instantaneous flow: (1) The upstream section is characterized by the mixing layer, with a rich coherent structure dynamics, that is mainly influenced by vortex shedding of the upstream jet and the shear layer between the SSZ and the channel; and (2) the downstream part of the 

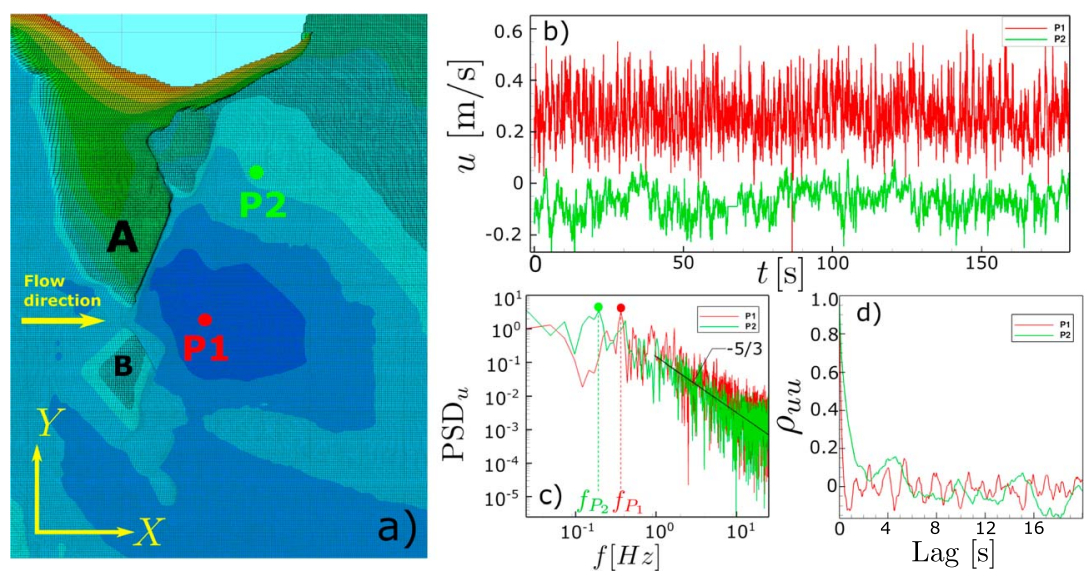

Figure 12. Time series and statistics of streamwise velocity in two points of the surface storage zone system measured with the acoustic Doppler velocimeter. (a) Location of points P1 and P2, where the velocity was measured at $0.05 \mathrm{~m}$ below the free surface; (b) time series of streamwise velocity $(u)$, showing clearly the differences on their magnitude and frequency; (c) power spectra $\left(P S D_{u}\right)$ show that the leading frequencies $(f)$ of the velocity at the interface and inside the surface storage zone are harmonics; and (d) autocorrelation functions of velocity $\left(\rho_{u u}\right)$ show the time-dependent structure of the flow, with longer time scales inside the cavity.

mixing layer, where the dominant coherent structures are streamwise vortices with spanwise rotation, that are stretched and separated as they move upward and approach the impingement point at the wall.

These vortices play a significant role on the flow patterns observed at the free surface with LSPIV. As they move upward, they attach to the free-surface, producing intermittent "boils" or upwelling events. To provide additional insights on the rotational dynamics of these vortical structures, in Figure 11, we plot a sequence of instantaneous $q$ isosurfaces colored by the sign of the local helicity of the flow. The helicity $\mathcal{H}$ is calculated from the resolved velocity projected on the vorticity field as follows:

$$
\mathcal{H}=u_{i} \omega_{i}
$$

where $u_{i}$ and $\omega_{i}$ correspond to the instantaneous velocity and vorticity vectors, respectively. The second and third columns of Figure 11 are zoomed images of the coherent structures at the impingement zone and the instantaneous velocity field at the free surface colored by dimensionless vertical velocity, respectively. Through this analysis, we can clearly visualize the formation of upwelling events at the free surface, which occur in the region of the source point identified with LSPIV. A pair of counterrotating vortices is formed in the upslope section with high mean $Y$ vorticity shown in Figure 8b. These two vortices (V1 and V2) are advected downstream, as depicted in Figure 11a, and approach the impingement region. As indicated by the yellow arrows, the structures are stretched by the bifurcating flow, which is identified by the different signs of the helicity field. Figures 11b-11d show the effect on the increase of the upwelling effect (increase of vertical velocity magnitude) produced by the interaction of V1 and V2 when the are rotating together and attach to the free surface. Finally, as shown in Figure 11f, the vortices separate and lose coherence and the upwelling zone vanishes. The process is then reinitiated with the formation of new vortices that emerge from the adverse slope zone. The interactions of the flow with the bathymetry induce new complex vortex interactions and vertical flows that contribute to the mass and momentum exchange, in addition to the shear layer and recirculation observed in simplified geometries.

In general, these boils constitute an important mechanism for sediment and mass transport processes in riverine environments, producing redistribution of momentum, temperature, nutrients, and sediments, as shown in estuaries with abrupt changes of bathymetry (Chickadel et al., 2009). The presence of upwelling events has been widely reported as a macroturbulence structure with quasiperiodic behavior, which has also been related to the formation of dunes in rivers (Best, 2005). In our case, the bathymetry gradient along interfacial zone generates an upslope similar to the upstream face of a dune, and the flow advects these coherent structures downstream to the impinging point at the free surface. Arsenic-laden particles can therefore be resuspended and transported by vortices of the shear layer near the free surface, a mechanism that is not accounted for in many traditional modeling approaches. 


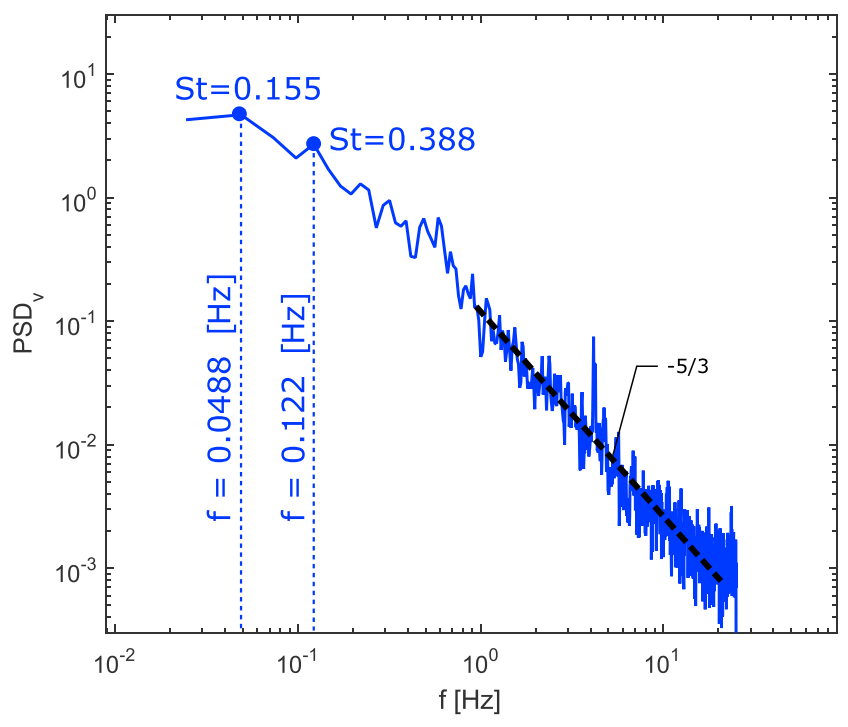

Figure 13. Cross-stream (transverse) velocity spectrum $\left(P S D_{v}\right)$ at point $\mathrm{P} 1$, defined in Figure 12, shows low-frequency components that are also harmonics. Two frequency peaks are identified in spectral analysis with their corresponding Strouhal numbers $(S t)$.
The dominant time scales of the flow at the interface are obtained from the analysis of the ADV velocity time series and their spectra. In Figure 12, we show the time series of two streamwise velocity components, at two different points within the cavity. These points are located $5 \mathrm{~cm}$ below the water surface level. At point $\mathrm{P} 1$, the streamwise velocity is positive toward downstream with high-frequency fluctuations, while at point P2, inside the SSZ, the streamwise velocity is mostly negative toward the upstream direction, with a smaller magnitude, lower frequency fluctuations, and sporadic crossings to positive values. These phenomena can be observed in more detail in the frequency analysis shown in Figures 12c and 12d, where we plot the velocity spectra and autocorrelation functions of the series, using a discrete estimation of these statistics (Pope, 2000). These show that there is one leading periodic process associated with the flow, with a higher frequency in P1 compared to P2. The velocity spectra also show that the frequencies associated to the peaks, $f_{P_{1}}=0.3662 \mathrm{~Hz}$ and $f_{P_{2}}=0.1221 \mathrm{~Hz}$, are harmonics as $f_{P_{1}} / f_{P_{2}}=3$, related to the vortex shedding at the interface.

To identify the leading frequency of vortex shedding at the interface, we plot in Figure 13 the measured transverse velocity power spectra of P1. In this spectrum we can also identify two predominant modes: The first one corresponds to a frequency of $0.0488 \mathrm{~Hz}$, while the second one corresponds to $0.1221 \mathrm{~Hz}$ with a ratio of 2.5 . In nondimensional terms, these frequencies correspond to Strouhal numbers $\left(S t=f L / U_{b}\right)$ of 0.155 and 0.388 , respectively. The first low-frequency mode is in the range reported by Liu and Katz (2013) for the slow flapping of the mixing layer, in a similar aspect ratio cavity. For the second leading frequency, the Strouhal number is consistent with the vortex shedding frequencies obtained by Jackson et al. (2015), for several study cases in semicircular, backward conic, and forward conic cavities for similar aspect ratio and Reynolds numbers.

To explore more in detail the temporal characteristics of transverse velocity fluctuations along the mixing layer, we plot in Figure 14 the autocorrelation functions of five computed cross-stream velocities and their power spectra density along the interface, at $5 \mathrm{~cm}$ below the water surface. As seen in Figure 14a, the points located near of the leading edge of the SSZ exhibit autocorrelation functions dominated by processes with short periods (higher frequencies), coming from the jet-like flow. As we move along the mixing layer, the transverse velocity shows increasing dominant periods. These phenomena are observed in the spectra, as closer to the cavity leading edge the flow is dominated by higher-frequency processes from the vortex shedding and interactions of coherent structures from the mixing layer and the wake behind the in-stream boulder. As we move downstream, along the mixing layer, the coherent structures interact and develop producing a flow with larger vortices. This can also be verified in Figure 14b, where the three downstream points $(x / L=0.5, x / L=0.67$, and $x / L=0.84)$ show a common frequency peak at $0.39 \mathrm{~Hz}$.
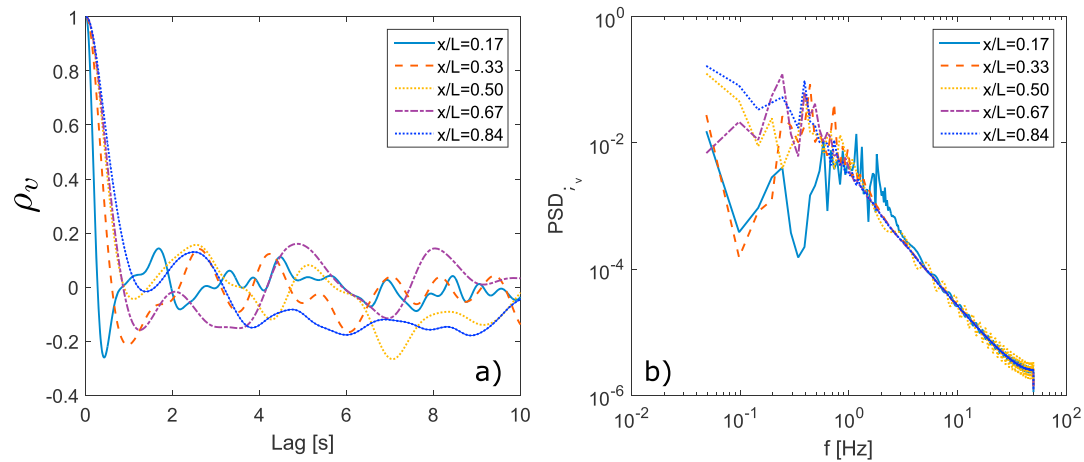

Figure 14. Transverse velocity statistics of five points computed with detached-eddy simulation along the interface, at $0.05 \mathrm{~m}$ below the free surface. (a) Autocorrelation functions $\left(\rho_{v}\right)$ and (b) power spectral density $\left(P S D_{\rho_{v}}\right)$ along the mixing layer $(x / L=0.17-0.84) 5 \mathrm{~cm}$ below the free surface. 


\section{Mass Transport}

The large-scale coherent structures of the mixing layer at the interface are the fundamental mechanism of mass exchange between the main channel and the cavity (Mignot et al., 2016). As described previously, their unsteady behavior is characterized by a periodic or quasiperiodic alternation of positive and negative fluctuations of transverse velocity, which drive mass and momentum exchange. The mass transport dynamics obtained from the DES numerical simulations shows the interplay of the coherent structures at the interface and the SSZ, including the effects of the intermittent upwelling events at the trailing edge of the cavity.

The collective dynamics of these coherent structures and their interactions with the recirculating region inside the SSZ are typically analyzed by using upscaled global models that aim to account for the exchange processes in predictions over larger scales. In this work we employ the DES results to investigate the evolution of the mean concentration in the SSZ and upscale the concentration field to fit three different 1-D models that represent the interaction of the cavity with the main channel at larger scales.

Two descriptors are most commonly used to quantify the mass exchange between the main channel and the lateral cavity (Jackson et al., 2013). The first one is a linear mass-exchange or nondimensional exchange coefficient $(k)$, defined as the ratio between the bulk velocity and the exchange velocity scale $(E)$. This velocity scale $E$ is the typical time and space averaged exchange velocity across the interface (Mignot et al., 2016). Valentine and Wood (1977) proposed $k$ as a constant, relating the bulk and exchange velocities as $E=k U_{b}$. The second method to quantify the exchange corresponds to the mean residence time of the cavity. Langmuir (1908) originally formulated a definition for the mean hydraulic residence time as the ratio of the cavity volume to the volumetric flow rate entering or leaving through the interface, along the shear layer (Jackson et al., 2013). Uijttewaal et al. (2001) combined the Langmuir (1908) definition with the entrainment hypothesis of Valentine and Wood (1977) to derive a predictive mean residence time $(\tau)$ relationship, given by the following expression:

$$
\tau=\frac{W L d_{c}}{k U L d_{E}}=\frac{W d_{c}}{E d_{E}}
$$

where $W$ and $L$ correspond to the cavity width and length, respectively; $d_{c}$ is the mean cavity depth; and $d_{E}$ is the mean shear-layer depth. This relation has the following four additional assumptions: (1) The flow is steady; (2) a residence time distribution exhibits a monotonic decay with time; (3) the exchange mechanism is a linear first-order process; and (4) the exchange coefficient $k$ is a nondimensional constant (Jackson et al., 2013).

This 1-D global model can therefore be employed to quantify the mass transport dynamics and exchange processes between the main channel and the SSZ, predicting the evolution of the mean concentration over time. O'Connor et al. (2010) expressed the linear single transient storage model (STSM) for SSZs as follows:

$$
\frac{\mathrm{d} C}{\mathrm{~d} t}=-\frac{E}{W} \frac{d_{\mathrm{m}}}{d_{c}}\left(C(t)-C_{\mathrm{m}}\right)=-\lambda\left(C(t)-C_{\mathrm{m}}\right),
$$

where $C_{m}$ and $d_{m}$ are the mean concentration and mean depth in the main channel, respectively. We can calculate the exchange velocity $E$ from the transverse velocity at the interface, following the methodology of Weitbrecht et al. (2008; see also Mignot et al., 2016; Tuna et al., 2013):

$$
E=\frac{1}{2 S_{e x}} \int_{S_{e x}} \overline{|v(x, z)|} \mathrm{d} S
$$

where $S_{e x}$ is the total area of the interface section. The value of the exchange coefficient $k$ is then obtained as the ratio between velocity exchange scale and the bulk velocity, $k=E / U_{b}$. In this case, the mass exchange coefficient yields a value of $k=0.0298$, which is in good agreement with the range of values reported in the literature for cavity flows. Equation (11) is a first-order transient storage model for the concentration in the cavity, and if $\lambda$ is considered as a constant, it can be integrated in time to obtain $C(t)$. 

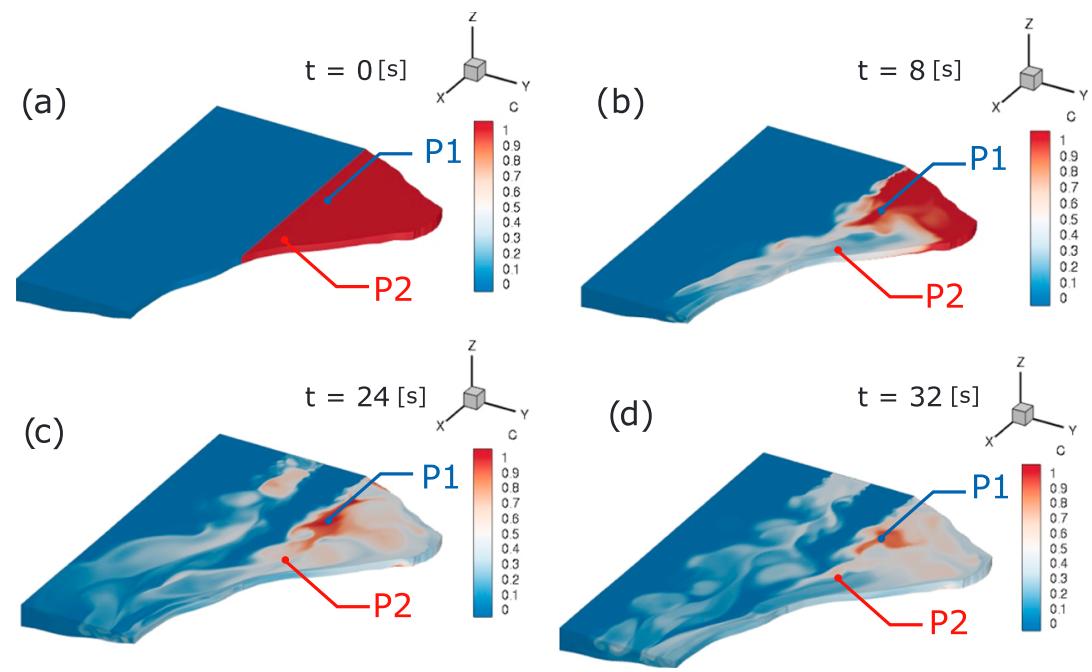

(e)

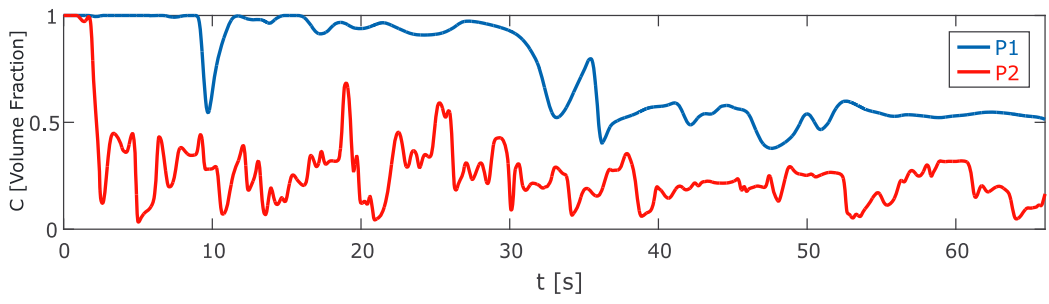

Figure 15. (a) to (d) show a sequence of images with the time evolution of the local solute concentration $C(t)$ in the main channel and the surface storage zone for different times. P1 is located in the inner region of the surface storage zone, meanwhile P2 is located in the outer region. (e) illustrates the time evolution of the concentration in these two points, which are located in zones with different exchange time scales, showing the influence of the mixing layer transport and the solute trapped in the primary vortex region.

By considering the dynamic response of the volume inside the SSZ, we can formulate an alternative model to describe the time evolution of the mean concentration within the entire cavity. A two-transient storage model (TTSM) considers the cavity volume by dividing it into two regions: (1) an inner region, which is approximately defined by the zone of influence of the primary vortex identified from limiting streamlines of the time-averaged velocity field; and (2) an outer region of the SSZ, which corresponds to the volume surrounding the inner region. The inner region can only exchange mass with the outer region. Meanwhile, the outer region can exchange mass with the inner one and the main channel, forming two transient storage zones. Assuming a first-order exchange rate for all of these processes, the conservation equations for each of these regions now depend on three parameters (instead of only $k$ ), and the model can be written as

$$
\begin{gathered}
\frac{\mathrm{d} C_{2}}{\mathrm{~d} t}=\beta\left(C_{1}(t)-C_{2}(t)\right), \\
\phi \frac{\mathrm{d} C_{1}}{\mathrm{~d} t}=\beta\left(C_{2}(t)-C_{1}(t)\right)-\alpha C_{1}(t) .
\end{gathered}
$$

In equations (13) and (14), $C_{1}$ corresponds to the concentration in the outer region, while $C_{2}$ is the concentration of the inner region. The parameter $\beta$ is the exchange rate between the inner and outer regions, $\alpha$ is the exchange rate from the outer region to the main channel, $\phi=V_{1} / V_{2}$ is the ratio of the volumes of the two regions, $V_{1}$ is the volume of the outer region, and $V_{2}$ is the volume of the inner region comprised by the main vortex inside the SSZ. To study the exchange processes, we select an initial condition of constant and uniform concentration within the cavity. Under these conditions, the system of equations given by (13) and (14) has an analytical solution: 


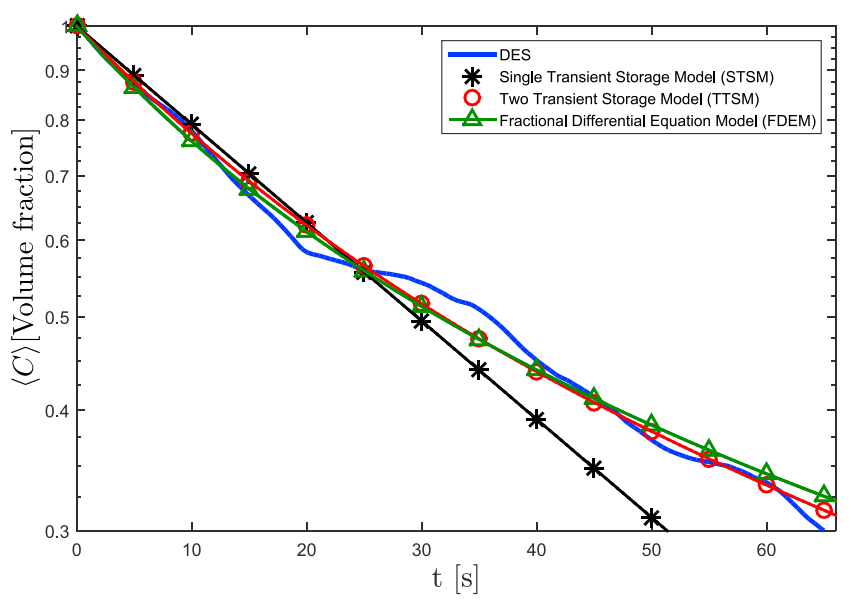

Figure 16. Comparison of spatially averaged concentration $(\langle C(t)\rangle)$ obtained from the high-resolution DES and simplified 1-D transport models for the evolution of the concentration in the surface storage zone. DES $=$ detached-eddy simulation.

$$
\begin{gathered}
C_{1}(t)=C_{0} e^{-t / 2(\alpha+\beta+\beta / \phi)}\left[\frac{\cosh \left(\frac{t}{2} \chi\right)}{\phi}+\frac{\sinh \left(\frac{t}{2} \chi\right)(\alpha \phi+\beta \phi+\beta)}{\phi \chi}\right], \\
C_{2}(t)=C_{0} e^{-t / 2(\alpha+\beta+\beta / \phi)}\left[\frac{\cosh \left(\frac{t}{2} \chi\right)}{\phi}+\frac{\sinh \left(\frac{t}{2} \chi\right)(-\alpha \phi+\beta \phi+\beta)}{\phi \chi}\right],
\end{gathered}
$$

where

$$
\chi=\sqrt{\alpha^{2} \phi^{2}+2 \alpha \beta \phi^{2}+\beta^{2} \phi^{2}-2 \alpha \beta \phi+2 \beta^{2} \phi+\beta^{2}} .
$$

The evolution of the mean concentration in the SSZ, computed with this 1-D TTSM, can be obtained as

$$
C(t)=\frac{\phi C_{2}(t)+C_{1}(t)}{\phi+1} .
$$

The advantage of using this two-storage model is the ability of representing different exchange rate time scales within the cavity, coupling the dynamics of two different regions within the volume. This can be a very important feature, as it allows us to explicitly identify the exchange time scales of the inner region, which might be much larger than the outer region that is exposed to the vortices of the shear layer.

Finally, we also consider a first-order fractional differential equation model (FDEM) to describe the time evolution of mean concentration in the cavity. Similar to the first 1-D model, this two-parameter model considers that the process can be represented by an ordinary linear differential equation, but in this case with a fractional order, as follows:

$$
\frac{\mathrm{d}^{\psi} C}{\mathrm{~d} t^{\psi}}=-k C(t)
$$

where $\psi$ corresponds to the order of the fractional derivative $(0<\psi<1)$, and $k$ is the same nondimensional exchange coefficient at larger scales described previously for the linear model.

For an initial condition $C(t=0)=C_{0}$, the solution of the first-order fractional differential equation is

$$
C(t)=C_{0} E_{\psi}(-k t)
$$

where $E_{\psi}$ is the Mittag-Leffer function, which is a generalization of the exponential function (Podlubny, 1998), defined as

$$
E_{\psi}(z)=\sum_{n=0}^{\infty} \frac{z^{n}}{\Gamma(1+n \psi)},
$$

where $z$ is the complex argument, and $\Gamma$ is the Gamma function. Time fractional equations typically reflect memory effects, which can capture complex internal dynamics (such as heavy tailed waiting time processes)

Table 4

Adjusted Parameters and Values of the Transient Storage Models

\begin{tabular}{lccc}
\hline Model & Adjusted parameters & Adjusted values & $R M S E$ \\
\hline STSM & $\lambda$ & 0.0235 & 0.0556 \\
TTSM & $\alpha, \beta, \phi$ & $0.0422,0.0061,0.532$ & 0.0170 \\
FDEM & $\psi$ & 0.11 & 0.0170 \\
\hline
\end{tabular}

Note. STSM $=$ single transient storage model; TTSM $=$ two-transient storage model; FDEM = fractional differential equation model. 
in effective manner, with a parsimonious model that only requires limited parameterization. The memory effect of the 1-D process decreases as $\psi \rightarrow 1$ (Sun et al., 2011).

In Figure 15, we show the evolution of $C(t)$ at two different points within the SSZ computed by DES. The solute concentration in a point inside the primary vortex identified in the mean flow field shows a slower decay compared to the outside point, which is located near the reattachment point, at the downstream end of the shear layer. These features provide the physical justification of the two-storage model and of the long memory fractional model that we presented above-that is, a single mean concentration is not representative of the concentration within the SSZ.

We compute the time evolution of the spatial average concentration within the cavity using the instantaneous results of the numerical simulation as follows:

$$
\langle C(t)\rangle=\frac{1}{V_{S S Z}} \int_{\Omega_{S S Z}} C(t) \mathrm{d} V,
$$

where $\langle C(t)\rangle$ is the volume-averaged concentration within the SSZ.

In Figure 16, we plot the instantaneous volume-averaged concentration and the concentrations using the three transient storage models: STSM, TTSM, and FDEM. The parameters for each model are fitted by minimizing the RMSE (Root mean square error), shown in Table 4. The results show that the time evolution of the SSZ-averaged concentration exhibits a heavy-tailing behavior with an apparent change of the decay rate, somewhere around 20 to $40 \mathrm{~s}$. This rate change is related to the time scale when most of the contaminant in the region surrounding the primary vortex has been transported out to the main channel, and higher solute concentrations remain only in the region of the primary structure.

Even though the STSM shows good agreement with the data at short time scales, it deviates as time progresses. A single storage zone model cannot represent such a regime change, which reflects the turbulent coherent structures interacting and increasing the residence time of the solute in some regions of the SSZ. On the other hand, both the TTSM and FDEM exhibit a good fit over the full range of simulated time scales, although some differences arise in the transitional intermediate section of the plot. In spite of these differences, the TTSM and FDEM show much better agreement compared to STSM (RMSE of 0.017 for both the TTSM and FDEM vs. 0.0556 for the STSM). FDEM presents an advantage, since it is defined by just one parameter, compared to the three parameters that define the TTSM (additional to $k$ in both cases). The small fractional exponent $\psi=0.11$ in the FDEM highlights the long-term correlation of the mass exchange processes within the cavity, which is a consequence of the interacting coherent structures of the flow.

\section{Conclusions and Future Work}

In this work we carried out a field and numerical investigation of a natural SSZ in the Lluta River, located in northern Chile, impacted by legacy acid mine drainage and the transport of arsenic-laden particles. We focus on the effects of turbulent coherent structures produced in the SSZ. Specifically, we study the shear layer and the recirculation zone dynamics, which are the physical mechanisms that control the ultimate fate of pollutants from acid-mine drainage and their interactions with the local environment.

The use of different field approaches is critical for characterizing the flow in natural conditions as they provide the necessary input further analyze the results of the numerical simulations. Through the combination of three measurement techniques and Detached-Eddy Simulation (DES) numerical modeling, we quantitatively described the most important features of the flow and their impacts on solute transport.

We measured the velocity at several points along the interface between the lateral recirculating region and the main channel using an acoustic Doppler velocimetry (ADV) device, and using a large-scale particle image velocimetry LSPIV technique, we reconstructed the time-averaged flow at the free surface. From photos of the riverbed and employing precise georeferenced locations, we obtained a DEM of the SSZ and the adjacent section of the channel, which was used to perform the numerical simulations.

Through a comparative analysis of the mean and instantaneous field data and the results of the numerical simulations, we characterized the flow field, identifying three important interacting mechanisms: (1) the complex vortex shedding of the shear layer, which oscillates continuously at the interface between the SSZ and the main channel producing an intense mass exchange; (2) the formation of counterrotating pairs of vortices near the bed, in the adverse slope region, which are advected and stretched as they approach the 
free surface near the impinging point of the shear layer, creating intermittent upwelling or "boiling" events; and (3) a large and stable recirculating region inside the cavity, which traps mass and produces long residence times and reduced exchange with the main channel. Importantly, these three processes interact and influence the resuspension, deposition, and downstream transport of mass in the river.

Finally, from the 3-D concentration field that we obtained from the numerical simulation of the SSZ passive scalar emptying process, we analyzed global mass transport associated with the cavity at larger spatial and temporal scales. We investigated the evolution of the average concentration within the SSZ through integrated 1-D models, comparing the classical linear transport model (STSM) with a physically motivated two-storage formulation (TTSM) and a fractional transport approach (FDEM). The TTSM and FDEM formulations captured better the temporal evolution of mean concentration in the cavity than the STSM. Particularly, these two models were capable of capturing the changes in the decay rate, which are related to the time when most of the solute in the region surrounding the primary vortex has been transported to the main channel, and higher solute concentrations only remain in the primary vortex region. The advantage of the FDEM is that it only employs two parameters: the linear exchange coefficient and the fractional exponent that reflects the memory of the system induced by the turbulent coherent structures of the flow. Through this research, we also tested the applicability and the scope of both traditional and new 1-D models to predict the evolution of passive contaminants in the SSZ from a global perspective.

In future research, we will work on improving these global descriptions by calculating the parameters of the 1-D models from geometric characteristics of the SSZ and nondimensional parameters of the flow. We will also investigate the efficiency of contaminant transport and downstream effects of SSZs in series along the river channel and analyze the consequences of contaminant transport during extreme flood events, which can change the Reynolds and Froude numbers of the flow, produce erosion, and even overflow the SSZ.

Acknowledgments

This work has been supported by

Fondecyt grant 1130940 . C.E. and L.M. acknowledge the funding from CONICYT/FONDAP grant 15110017. This research was partially supported by the supercomputing infrastructure of the NLHPC (ECM-02). We thank Olivia K. August for motivating the global description of mass transport. We greatly appreciate Bruce MacVicar, Paul Grams, and two anonymous reviewers for their contributions to improve the quality of this paper. The data from field measurements is available for download in the following open data repository (https://doi.org/ 10.5281/zenodo.1345310).

\section{References}

Abarca, M., Guerra, P., Arce, G., Montecinos, M., Escauriaza, C., Coquery, M., \& Pastén, P. (2017). Response of suspended sediment particle size distributions to changes in water chemistry at an Andean mountain stream confluence receiving arsenic rich acid drainage. Hydrological Processes, 31(2), 296-307. https://doi.org/10.1002/hyp.10995

Alvarez, L. V., Schmeeckle, M. W., \& Grams, P. E. (2017). A detached eddy simulation model for the study of lateral separation zones along a large canyon-bound river. Journal of Geophysical Research: Earth Surface, 122, 25-49. https://doi.org/10.1002/2016JF003895

Arce, G., Montecinos, M., Guerra, P., Escauriaza, C., Coquery, M., \& Pastén, P. (2017). Enhancement of particle aggregation in the presence of organic matter during neutralization of acid drainage in a stream confluence and its effect on arsenic immobilization. Chemosphere, 180, 574-583. https://doi.org/10.1016/j.chemosphere.2017.03.107

Bencala, K. E., \& Walters, R. A. (1983). Simulation of solute transport in a mountain pool-and-rifle stream: A transient storage model. Water Resources Research, 19(3), 718-724.

Best, J. (2005). The fluid dynamics of river dunes: A review and some future research directions. Journal of Geophysical Research, 110, F04S02. https://doi.org/10.1029/2004JF000218

Butler, B. A., Ranville, J. F., \& Ross, P. E. (2008). Observed and modeled seasonal trends in dissolved and particulate Cu, Fe, Mn, and Zn in a mining-impacted stream. Water Research, 42(12), 3135-3145. https://doi.org/10.1016/j.watres.2008.03.004

Chang, K., Constantinescu, G., \& Park, S. O. (2006). Analysis of the flow and mass transfer processes for the incompressible flow past an open cavity with a laminar and a fully turbulent incoming boundary layer. Journal of Fluid Mechanics, 561, 113-145. https://doi.org/ $10.1017 /$ S0022112006000735

Chickadel, C. C., Horner-Devine, A. R., Talke, S. A., \& Jessup, A. T. (2009). Vertical boil propagation from a submerged estuarine sill. Geophysical Research Letters, 36, L10601. https://doi.org/10.1029/2009GL037278

Constantinescu, G., Sukhodolov, A., \& McCoy, A. (2009). Mass exchange in a shallow channel flow with a series of groynes: LES study and comparison with laboratory and field experiments. Environmental Fluid Mechanics, 9(6), 587-615. https://doi.org/10.1007/ s10652-009-9155-2

Contreras, M. T., Müllendorff, D., Pastén, P., Pizarro, G. E., Paola, C., \& Escauriaza, C. (2015). Potential accumulation of contaminated sediments in a reservoir of a high-Andean watershed: Morphodynamic connections with geochemical processes. Water Resources Research, 51, 3181-3192. https://doi.org/10.1002/2014WR016130

Coz, J. L., Hauet, A., Pierrefeu, G., Dramais, G., \& Camenen, B. (2010). Performance of image-based velocimetry (LSPIV) applied to flash-flood discharge measurements in Mediterranean rivers. Journal of Hydrology, 394(1), 42-52. https://doi.org/10.1016/ j.jhydrol.2010.05.049, flash Floods: Observations and Analysis of Hydrometeorological Controls.

Dietrich, J. T. (2017). Bathymetric structure-from-motion: Extracting shallow stream bathymetry from multi-view stereo photogrammetry. Earth Surface Processes and Landforms, 42(2), 355-364. https://doi.org/10.1002/esp.4060

Drost, K. J., Apte, S. V., Haggerty, R., \& Jackson, T. (2014). Parameterization of mean residence times in idealized rectangular dead zones representative of natural streams. Journal of Hydraulic Engineering, 140(8), 04014035. https://doi.org/10.1061/(ASCE)HY. 1943-7900.0000879

Escauriaza, C., \& Sotiropoulos, F. (2011a). Lagrangian model of bed-load transport in turbulent junction flows. Journal of Fluid Mechanics, 666, 3676. https://doi.org/10.1017/S0022112010004192

Escauriaza, C., \& Sotiropoulos, F. (2011b). Reynolds number effects on the coherent dynamics of the turbulent horseshoe vortex. Flow, Turbulence and Combustion, 86(2), 231-262. https://doi.org/10.1007/s10494-010-9315-y 
Escauriaza, C., \& Sotiropoulos, F. (2011c). Initial stages of erosion and bed form development in a turbulent flow around a cylindrical pier. Journal of Geophysical Research, 116(July), F03007. https://doi.org/10.1029/2010JF001749

Fujita, I., Muste, M., \& Kruger, A. (1998). Large-scale particle image velocimetry for flow analysis in hydraulic engineering applications Journal of Hydraulic Research, 36(May 2015), 397-414. https://doi.org/10.1080/00221689809498626

Garreaud, R., Vuille, M., \& Clement, A. C. (2003). The climate of the altiplano: Observed current conditions and mechanisms of past changes. Palaeogeography, Palaeoclimatology, Palaeoecology, 194(1), 5-22. https://doi.org/10.1016/S0031-0182(03)00269-4, late-quaternary palaeoclimates of the southern tropical Andes and adjacent regions.

Guerra, P., Gonzalez, C., Escauriaza, C., Pizarro, G., \& Pasten, P. (2016). Incomplete mixing in the fate and transport of arsenic at a river affected by acid drainage. Water, Air, \& Soil Pollution, 227(3), 73.

Guerra, P., Simonson, K., González, C., Gironás, J., Escauriaza, C., Pizarro, G., et al. (2016). Daily freeze-thaw cycles affect the transport of metals in streams affected by acid drainage. Water, 8(3), 74.

Hudson-Edwards, K. (2003). Sources, mineralogy, chemistry and fate of heavy metal-bearing particles in mining-affected river systems.

Hudson-Edwards, K. (2016). Tackling mine wastes. Science, 352(6283), 288-290. https://doi.org/10.1126/science.aaf3354

Hunt, J. C. R., Wray, A. A., \& Moin, P. (1988). Eddies, stream, and convergence zones in turbulent flows. In Proceedings of the Summer Program. Center for Turbulence Research (pp. 193-208). NASA Ames/Stanford University.

Jackson, T. R., Apte, S. V., Haggerty, R., \& Budwig, R. (2015). Flow structure and mean residence times of lateral cavities in open channel flows: Influence of bed roughness and shape. Environmental Fluid Mechanics, 15(5), 1069-1100. https://doi.org/10.1007/ s10652-015-9407-2

Jackson, T. R., Haggerty, R., Apte, S. V., Coleman, A., \& Drost, K. J. (2012). Defining and measuring the mean residence time of lateral surface transient storage zones in small streams. Water Resources Research, 48, W10501. https://doi.org/10.1029/2012WR012096

Jackson, T. R., Haggerty, R., Apte, S. V., \& O'Connor, B. L. (2013). A mean residence time relationship for lateral cavities in gravel-bed rivers and streams: Incorporating streambed roughness and cavity shape. Water Resources Research, 49, 3642-3650. https://doi.org/ 10.1002/wrcr.20272

Julien, P. Y. (2010). Erosion and sedimentation. Cambridge: Cambridge University Press.

Kantoush, S. A., Schleiss, A. J., Sumi, T., \& Murasaki, M. (2011). LSPIV implementation for environmental flow in various laboratory and field cases. Journal of Hydro-environment Research, 5(4), 263-276. https://doi.org/10.1016/j.jher.2011.07.002

Khosronejad, A., Hansen, A. T., Kozarek, J. L., Guentzel, K., Hondzo, M., Guala, M., et al. (2016). Large eddy simulation of turbulence and solute transport in a forested headwater stream. Journal of Geophysical Research: Earth Surface, 121, 146-167. https://doi.org/10.1002/ 2014JF003423

Kimura, I., \& Hosoda, T. (1997). Fundamental properties of flows in open channels with dead zone. Journal of Hydraulic Engineering, 123(August), 98-107. https://doi.org/0.1061/(ASCE)0733-9429(1997)123:2(98)

Knapp, J. L. A., Gonzlez-Pinzn, R., Drummond, J. D., Larsen, L. G., Cirpka, O. A., \& Harvey, J. W. (2017). Tracer-based characterization of hyporheic exchange and benthic biolayers in streams. Water Resources Research, 53, 1575-1594. https://doi.org/10.1002/2016WR019393

Kossoff, D., Dubbin, W. E., Alfredsson, M., Edwards, S. J., Macklin, M. G., \& Hudson-Edwards, K. A. (2014). Mine tailings dams: Characteristics, failure, environmental impacts, and remediation. Applied Geochemistry, 51, 229-245. https://doi.org/10.1016/ j.apgeochem.2014.09.010

Laity, J. J. (2008). Deserts and desert environments (2 pp.). Chichester, West Sussex, UK: John Wiley.

Langmuir, I. (1908). The velocity of reactions in gases moving through heated vessels and the effect of convection and diffusion. Journal of the American Chemical Society, 30(11), 1742-1754. https://doi.org/10.1021/ja01953a011

Leiva, E., Rámila, C., Vargas, I., Escauriaza, C., Bonilla, C., Pizarro, G., et al. (2014). Natural attenuation process via microbial oxidation of arsenic in a high Andean watershed. Science of The Total Environment, 467, 490-502.

Leonard, B. P. (1991). The ultimate conservative difference scheme applied to unsteady one-dimensional advection. Computer Methods in Applied Mechanics and Engineering, 88(1), 17-74. https://doi.org/10.1016/0045-7825(91)90232-U

Link, O., González, C., Maldonado, M., \& Escauriaza, C. (2012). Coherent structure dynamics and sediment particle motion around a cylindrical pier in developing scour holes. Acta Geophysica, 60(6), 1689-1719. https://doi.org/10.2478/s11600-012-0068-y

Liu, X., \& Katz, J. (2013). Vortex-corner interactions in a cavity shear layer elucidated by time-resolved measurements of the pressure field. Journal of Fluid Mechanics, 728, 417-457. https://doi.org/10.1017/jfm.2013.275

Micheletti, N., Chandler, J. H., \& Lane, S. N. (2015). Structure from motion (SfM) photogrammetry. British Society for Geomorphology, 2(2), 1-12. https://doi.org/10.5194/isprsarchives-XL-5-W4-37-2015

Mignot, E., Cai, W., Launay, G., Riviere, N., \& Escauriaza, C. (2016). Coherent turbulent structures along the mixing-interface of a square open-channel lateral cavity. Physics of Fluids, 28, 1-15. https://doi.org/10.1063/1.4945264

Mignot, E., Cai, W., Polanco, J. I., Escauriaza, C., \& Riviere, N. (2017). Measurement of mass exchange processes and coefficients in a simplified open-channel lateral cavity connected to a main stream. Environmental Fluid Mechanics, 17(3), 429-448.

O'Connor, B. L., Hondzo, M., \& Harvey, J. W. (2010). Predictive modeling of transient storage and nutrient uptake: Implications for stream restoration. Journal of Hydraulic Engineering, 136(12), 1018-1032. https://doi.org/10.1061/(ASCE)HY.1943-7900.0000180

Ozalp, C., Pinarbasi, A., \& Sahin, B. (2010). Experimental measurement of flow past cavities of different shapes. Experimental Thermal and Fluid Science, 34(5), 505-515. https://doi.org/10.1016/j.expthermflusci.2009.11.003

Paik, J., Escauriaza, C., \& Sotiropoulos, F. (2007). On the bimodal dynamics of the turbulent horseshoe vortex system in a wing-body junction. Physics of Fluids, 19(4), 045107. https://doi.org/10.1063/1.2716813

Paik, J., Escauriaza, C., \& Sotiropoulos, F. (2010). Coherent structure dynamics in turbulent flows past in-stream structures: Some insights gained via numerical simulation. Journal of Hydraulic Engineering, 136(12), 981-993. https://doi.org/10.1061/(ASCE)HY 1943-7900.0000089

Patil, S., Covino, T. P., Packman, A. I., McGlynn, B. L., Drummond, J. D., Payn, R. A., \& Schumer, R. (2013). Intrastream variability in solute transport: Hydrologic and geomorphic controls on solute retention. Journal of Geophysical Research: Earth Surface, 118, 413-422. https://doi.org/10.1029/2012JF002455

Podlubny, I. (1998). Fractional differential equations: An introduction to fractional derivatives, fractional differential equations, to methods of their solution and some of their applications (Vol. 198). San Diego, CA: Elsevier.

Pope, S. B. (2000). Turbulent flows. Cambridge: Cambridge University Press.

Rodriguez-Freire, L., Avasarala, S., Ali, A.-M. S., Agnew, D., Hoover, J. H., Artyushkova, K., et al. (2016). Post Gold King Mine spill investigation of metal stability in water and sediments of the Animas River watershed. Environmental Science \& Technology, 50(21), 11,539-11,548 https://doi.org/10.1021/acs.est.6b03092

Schmidt, J. C. (1990). Recirculating flow and sedimentation in the Colorado River in Grand Canyon, Arizona. The Journal of Geology, 98(5), $709-724$ 
Shur, M., Spalart, P. R., Strelets, M., \& Travin, A. (1999). Detached-eddy simulation of an airfoil at high angle of attack. In W. Rodi \& D. Laurence (Eds.), Engineering turbulence modelling and experiments 4 (pp. 669-678). Oxford: Elsevier Science Ltd. https://doi.org/10.1016/B978-008043328-8/50064-3

Sotiropoulos, F., \& Constantinescu, G. (1997). Pressure-based residual smoothing operators for multistage pseudocompressibility algorithms. Journal of Computational Physics, 133(1), 129-145.

Spalart, P. R. (2009). Detached-eddy simulation. Annual Review of Fluid Mechanics, 41(1), 181-202. https://doi.org/10.1146/annurev.fluid. 010908.165130

Sun, H., Chen, W, Wei, H, \& Chen, Y. (2011). A comparative study of constant-order and variable-order fractional models in characterizing memory property of systems. The European Physical Journal Special Topics, 193(1), 185-192.

Tuna, B. A., Tinar, E., \& Rockwell, D. (2013). Shallow flow past a cavity: Globally coupled oscillations as a function of depth. Experiments in Fluids, 54(8), 20. https://doi.org/10.1007/s00348-013-1586-3

Uijttewaal, W. S. J., Lehmann, D., \& van Mazijk, A. (2001). Exchange processes between a river and its groyne fields: Model experiments. Journal of Hydraulic Engineering, 127(11), 928-936. https://doi.org/10.1061/(ASCE)0733-9429(2001)127:11(928)

Valentine, E. M., \& Wood, I. R. (1977). Longitudinal dispersion with dead zones. Journal of the Hydraulics Division, 103(9), 975-990.

Weitbrecht, V., \& Jirka, G. H. (2001). Flow patterns and exchange processes in dead zones of rivers. In Proceedings of the congress-international association for hydraulic research (pp. 439-435).

Weitbrecht, V., Kühn, G., \& Jirka, G. H. (2002). Large scale PIV-measurements at the surface of shallow water flows. Flow Measurement and Instrumentation, 13(5-6), 237-245. https://doi.org/10.1016/S0955-5986(02)00059-6

Weitbrecht, V., Socolofsky, S. a., \& Jirka, G. H. (2008). Experiments on mass exchange between groin fields and main stream in rivers. Journal of Hydraulic Engineering, 134(2), 173-183. https://doi.org/10.1061/(ASCE)0733-9429(2008)134:2(173)

Wilcox, A., Escauriaza, C., Agredano, R., Mignot, E., Zuazo, V., Otárola, S., et al. (2016). An integrated analysis of the 2015 Atacama floods. Geophysical Research Letters, 43, 8035-8043. https://doi.org/10.1002/2016GL069751 